Research Article

\title{
Preliminary Research on a High Thrust-to-Weight Ratio of Double-Sided Composite Impeller Microturbine Engine
}

\author{
Cheng Zhang, ${ }^{1}$ Bei Yang ${ }^{1},{ }^{1}$ Yun Wang, ${ }^{2}$ and Gaoxing Tu ${ }^{1}$ \\ ${ }^{1}$ School of Aircraft Engineering, Nanchang Hangkong University, Nanchang 330063, China \\ ${ }^{2}$ School of Intelligent Manufacturing, Taizhou University, Taizhou 318000, China \\ Correspondence should be addressed to Bei Yang; peipeiyang99@sina.com
}

Received 3 April 2021; Revised 1 August 2021; Accepted 1 November 2021; Published 1 December 2021

Academic Editor: Adel Ghenaiet

Copyright ( 92021 Cheng Zhang et al. This is an open access article distributed under the Creative Commons Attribution License, which permits unrestricted use, distribution, and reproduction in any medium, provided the original work is properly cited.

\begin{abstract}
In some situations, the improvement of the thrust-to-weight ratio (TWR) of microturbine engines (MTEs) for energy-, economic-, and environment-related reasons can be achieved for military or civilian purposes. However, due to limitations of existing traditional MTE technology, it is difficult to meet the key requirements of small aircraft for high energy/power density and low-cost power, especially for long-endurance drone swarms. To address these problems, a novel compact concept of a highTWR of MTE with a double-sided composite impeller (DSCI) is proposed in this research. First, the principle and structure of the concept are explained through theoretical analysis, and its potential advantages are discussed. Second, the DSCI is analyzed at the top level, and the design principle and important parameters are discussed. The DSCI and supporting jet engine are preliminarily designed. Then, their weight is estimated. Finally, theoretical analytical and numerical simulations are used to preliminarily research the performance parameters of DSCI jet engine at the design point, and the parameters are discussed. These calculations showed encouraging results, with all components of the DSCI jet engine meeting matching characteristics. Compared to the JetCat P500-PRO-GH, the DSCI jet engine has a 39.4\% increase in TWR and a $36.82 \%$ decrease in specific fuel consumption $(s f c)$. This study lays a foundation for the development of high energy/power density MTEs in the future.
\end{abstract}

\section{Introduction}

Reference [1] presented the main trends of research into microturbine engines. Currently, the MTE is usually defined as an engine with a thrust less than $100 \mathrm{daN}$ and mainly includes to military/civilian applications, such as the propulsion systems of small aircraft, the power of target missiles and cruise missiles, the portable energy of individual combat, and the auxiliary power unit (APU) of large aircraft [2-5]. The propulsion systems of this mechanical equipment have two important requirements, namely, long endurance and high energy/power density. As two important performance parameters, the thrust-to-weight ratio (TWR) and specific fuel consumption $(s f c)$ are related to the two requirements. Several traditional techniques, such as increasing the overall pressure ratio $(O P R)$, turbine inlet total temperature, and component efficiency, can improve the TWR and $s f c$. However, with the rapid development of aircraft performance, these parameters have recently faced technological limitations, and it is becoming less advantageous to increase the thermal efficiency and specific thrust for MTEs. However, reducing the weight of the structure, introducing impingement cooling technology, and using heat recovery technology are three appropriate methods for overcoming these limitations.

The traditional gas generator consists of a single-stage centrifugal compressor, an annular combustor, and a single-stage axial turbine. Based on the innovative idea that a compact structure can reduce the weight of MTEs, United Technologies Corporation has developed compact MTEs with back-to-back single-stage centrifugal compressor and radial turbine, such as the TJ-50, TJ-90, and other products, which have significantly improved TWR and are costeffective [6]. References [7-10] demonstrate that the current research hot spot is the pursuit of compact structures and high energy/power density MTEs.

From this perspective, using the idea of a compact structure and thermodynamic cycle fusion, along with metal 
additive manufacturing technology, a double-sided composite impeller (DSCI) and its application to the propulsion system are proposed. The DSCI consists of a double-sided centrifugal compressor and a hollow-tip turbine (turbine) as a potential solution for a high energy/power density MTE [11]. The turbine has the same number of blades as the centrifugal compressor.

Aerodynamic and thermodynamic analyses of such a DSCI and its propulsion system were conducted to verify its potential in increasing the TWR and turbine inlet total temperature. The performance parameters of the DSCI jet engine and all component matching characteristics were numerically simulated and theoretically analyzed through the RANS-based CFD method. As a demonstration, a typical MTE was selected as the prototype and compared with the DSCI jet engine. This study is expected to provide the necessary technical basis for the development of high-TWR microturbine engine technology in the future.

\section{Principle of the DSCI and Its Application to a High-TWR Propulsion System}

2.1. Principle and Structure of the DSCI. Figure 1 illustrates the structure of the DSCI. The DSCI consists of a doublesided centrifugal compressor driven by a hollow-tip turbine mounted at the double-sided centrifugal compressor tip. The hollow-tip turbine can achieve a positive circumferential velocity at a high spool speed. The two parts are attached to each other using a structural and aerodynamic design. The principle of the DSCI is shown in Figure 2 and is as follows. First, the centrifugal compressor breathes air from the atmosphere. Compressed air flows into the hollow-tip turbine inside and is further compressed using the principle of centrifugal supercharging. The high-pressure air is mixed with fuel in the reverse-flow annular combustor and combusted by a flame ignitor. Combusted hot gas expands in the hollow turbine guide and hollow-tip turbine outside, and the jet rotates at the hollow-tip turbine outlet. Rotary jet exhaust gives torque to the DSCI and supplies torque to the centrifugal compressor. The same process applies to the rear intake air. The blade numbers of the centrifugal compressor and the hollow-tip turbine must be the same to provide structural consistency and reliability.

The DSCI rotor consists of a double-sided centrifugal compressor (two centrifugal compressors are fixed back-toback), a hollow-tip turbine and two round hubs mounted at the double-sided centrifugal compressor tip, and a blade shroud located at the turbine tip. Through additive manufacturing technology, all parts of the DSCI rotor are manufactured in an integrated manner. The inside profile of the turbine is designed as a tubular diffuser structure. Additionally, the outside profile of the turbine maintains an axial turbine design.

2.2. Concept of the DSCI Jet Engine. DSCI jet engines are mainly applied to the power of missiles or target bombs. Figure 3 depicts a preliminary jet propulsion system that adapts the DSCI. The propulsion system primarily consists of four components: (1) a starting component; (2) a rotating

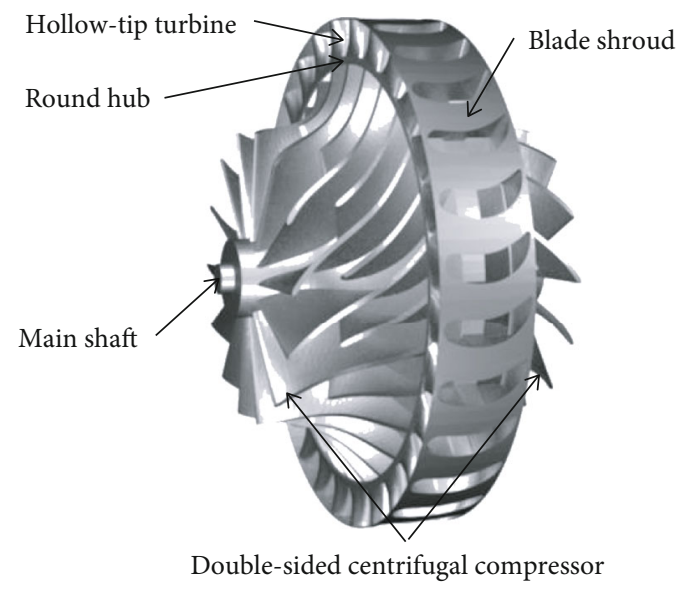

FIGURE 1: 3D model of the DSCI rotor.

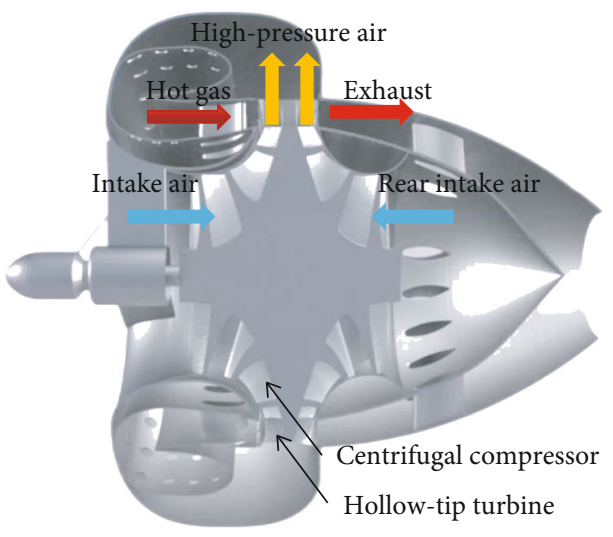

Figure 2: Principle of the DSCI rotor.

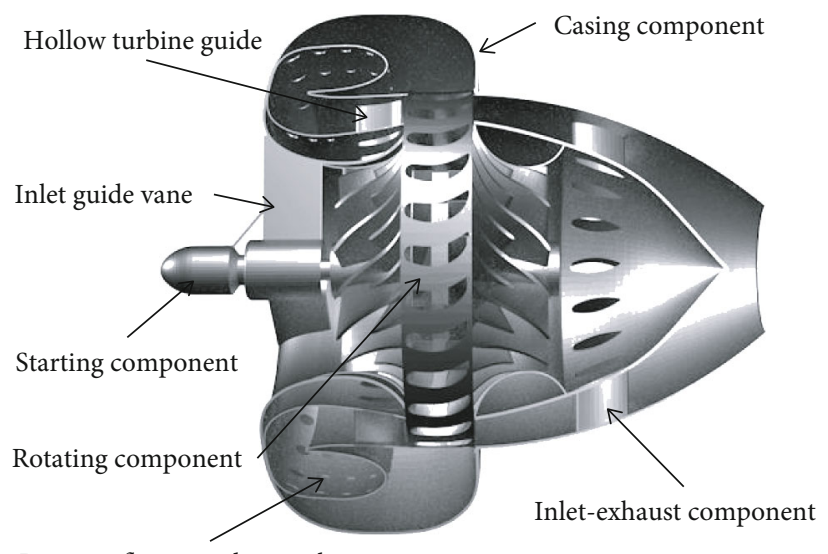

Reverse -flow annular combustor

FIgURE 3: Structure of the DCSI jet engine.

component; (3) a casing component; and (4) an inletexhaust component. Such a design has already been demonstrated in a Chinese patent [12].

As mentioned, the principle of the DSCI rotor is systematically analyzed. However, the principle of other components can be clarified as follows (Figure 4): 


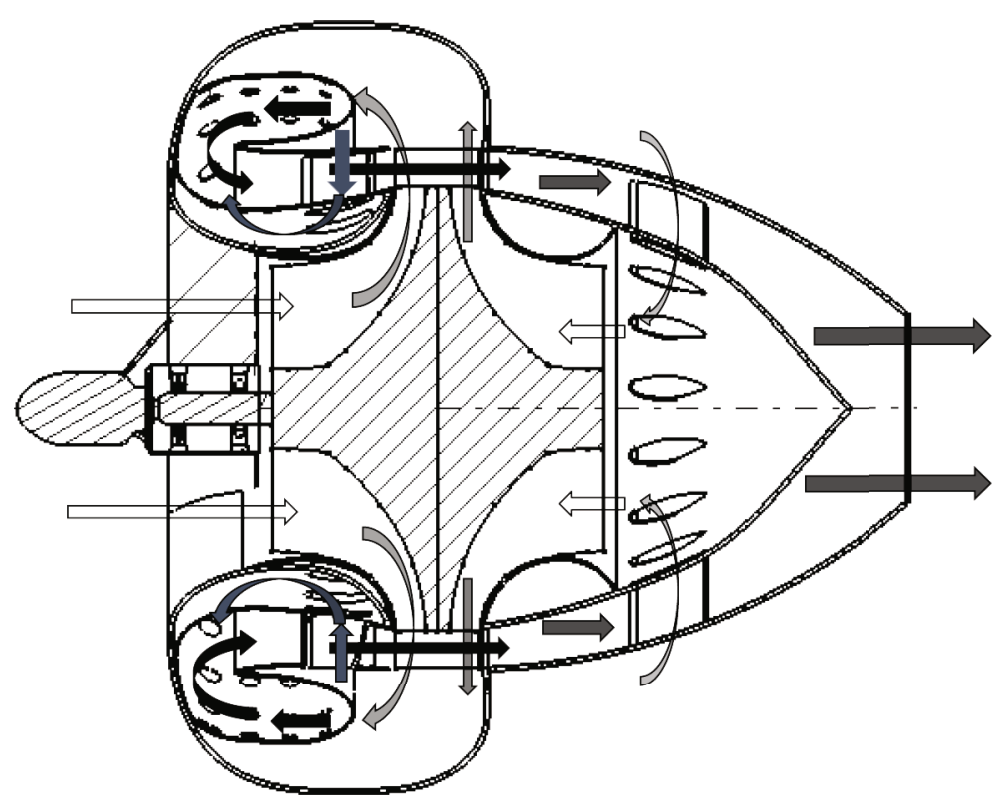

FIgUre 4: Principle of the DCSI jet engine [11].

(1) To spin up the DSCI rotor when the DSCI jet engine starts, the starting electric motor is connected to the DSCI by a magnetic clutch and main shaft. This motor can spin the DSCI up to $26,000 \mathrm{rpm}$. During spin up, the magnetic clutch transmits torque from the motor to the DSCI rotor. When spin up is completed, the clutch separates the motor and the DSCI rotor

(2) The high-pressure gas from inside the hollow-tip turbine is separated into two streams in the casing. The main part of the air directly flows into the reverse-flow annular combustor for stable combustion. The remaining air flows into the reverse-flow annular combustor after it exchanges heat with the high-temperature gas through the hollow turbine guide wall. This process prevents the reverse-flow annular combustor, prevents the turbine wall from overheating, and improves the life and reliability of the DSCI

(3) The DSCI jet engine uses a double fulcrum support method. The specific installation position requires that two bearings be installed to support the rotor components in the inlet guide vane.

2.3. Concept of the Gas-Driven Propeller DSCI Engine. The gas-driven propeller DSCI engine is a compact concept that further integrates a traditional turboprop engine with the DSCI jet engine and can be widely used to power expendable aircraft and drone swarms. Figure 5 shows a gasdriven propeller system that integrates the DSCI. The system includes 5 components: (1) a starting component; (2) a rotating component I (DSCI); (3) a casing component; (4) an inlet-exhaust component; and (5) a rotating component II (gas-driven propeller). Such a design has already been demonstrated in a Chinese utility model patent
[13]. For the concept of the gas-driven propeller DSCI engine, only a brief introduction is made here, and an in-depth study is not presented.

There are many similarities in principle and structure between the gas-driven propeller DSCI engine and the DCSI jet engine, but there are also some differences, which are described as follows:

(1) The gas-driven propeller is also an aerodynamic component like a DSCI with three parts: the root turbine, the hub, and the propeller. The root turbine is radially connected with the propeller through the hub. Unlike the DSCI, the gas-driven propeller is not connected to the main shaft but is mounted to the nozzle through two sets of thrust bearings. This is a bit like a shaftless pump, as shown in Figure 5

(2) To spin up the gas-driven propeller when the engine starts, the root turbine must be fixedly connected to the propeller through the hub. The root turbine is driven by the residual heat discharged from the DSCI rotor to provide power for the propeller. Meanwhile, owing to the torque balance, the root turbine plays a role similar to a reduction gearbox, namely, the aerodynamic reducer in Figure 6 [14]

(3) There are two rows of circumferential array rear intake pipes before and after the gas-driven propeller. The rear intake pipe, nozzle, and fairing cone are also integrated using additive manufacturing technology. Rear intake pipe I is located before the gas-driven propeller, which is located before rear intake pipe II as shown in Figure 5. In addition to satisfying some of the rear intake conditions, rear intake pipe I also plays an important role as the turbine guide for driving the root turbine. The 


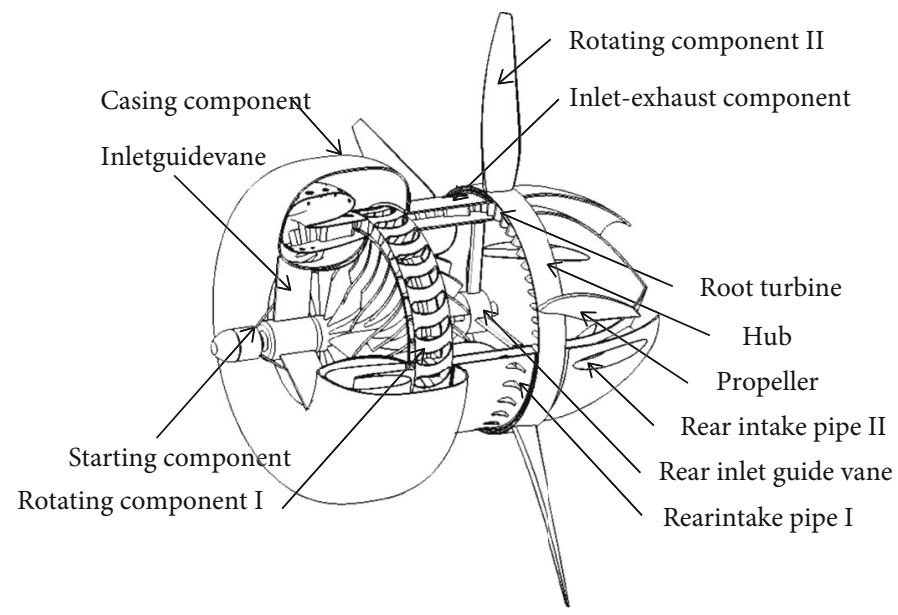

FIGURE 5: Structure of the gas-driven propeller DSCI engine.

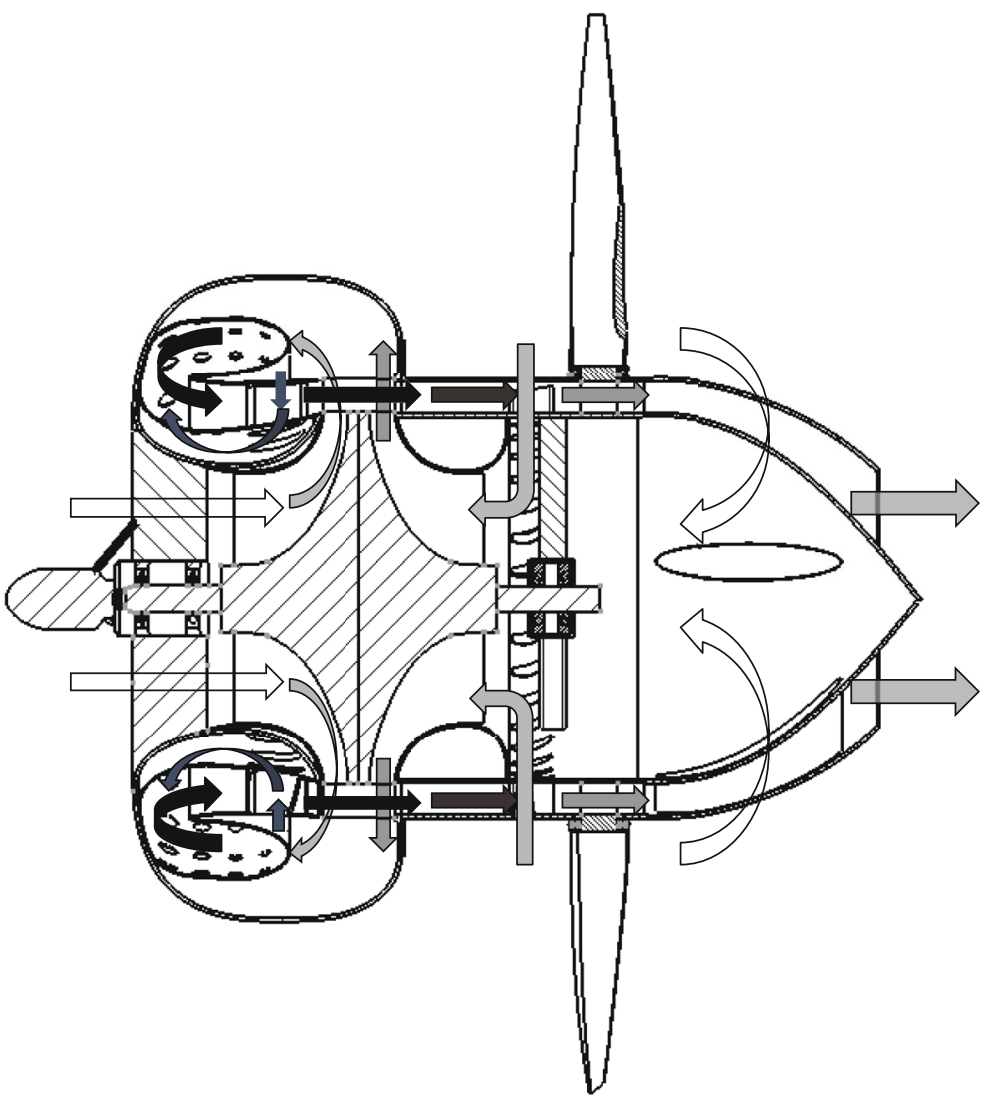

Figure 6: Principle of the gas-driven propeller DSCI engine.

profile of rear intake pipe II is designed to be elliptical, which not only meets the rear intake condition requirements but also actively guides the airflow at the outlet of the root turbine

(4) The gas-driven propeller DSCI engine adopts three fulcrum support schemes. Bearings are provided on the inlet guide vane and the rear inlet guide vane to support rotor component I (DSCI). Two rows of thrust bearings are arranged at the front and rear under the hub of the gas-driven propeller, and they are seamlessly connected with the nozzle to support the gas-driven propeller.

Through the preliminary analysis of the gas-driven propeller DSCI engine, the gas-driven propeller not only achieves power as a propulsion component but also replaces the reduction gearbox to achieve weight reduction as an aerodynamic reducer. Compared with the traditional shaft output power engine, there is not only a greater reduction 
in the axial size but also an obvious advantage in the structural weight. In addition, it is conducive to the reliability and starting characteristics of MTEs.

As has been mentioned, compared with the traditional MTEs, the DSCI engine has the following characteristics:

(1) Since the axial size of the DSCI engine has been greatly reduced, the coaxiality and shock absorption are good

(2) The DSCI adopts compact structure and thermal fusion characteristics, which not only cool the turbine but also recover exhaust heat. On the other hand, the DSCI improves the thermal efficiency of MTEs and provides for potential development of low-cost, lightweight materials, or increasing the turbine inlet total temperature

(3) The DSCI engine has a compact structure, which reduces the structural weight and significantly improves the $T W R / P W R$

(4) The DSCI engine adopts the fulcrum support scheme to keep the bearing away from the hightemperature environment, which is beneficial for improving the life and reliability of the bearing

(5) With the help of additive manufacturing technology, installation of a DSCI jet engine can be more convenient, and a reduction in the number of parts can make the structure more reliable.

\section{Preliminary Design of the DSCI and Its Jet Engine}

3.1. Preliminary Design Assumptions. To demonstrate the effects of DSCI, a preliminary design based on traditional MTEs. The first step for the preliminary design is to determine the top-level parameters of the DSCI jet engine. These parameters determine the performance of the DSCI jet engine and are used to guide the design of all components. According to thermodynamics theory, the performance parameters of the DSCI and its engine are approximately estimated if the following assumptions are introduced:

(1) For analysis convenience, the auxiliary impeller inlet conditions (the air breathed by centrifugal compressor through the rear intake pipe) are simplified to be the same as the main impeller inlet conditions

(2) Due to the complex structure of the reverse-flow annular combustor, it is simplified to an ideal state. The combustor outlet total temperature $T_{t 4}$ is $1150 \mathrm{~K}$. According to empirical coefficients and theoretical formulas, other cross-sectional parameters of the combustor can be obtained

(3) For analysis convenience, these parameters are set by default to be unchanged with static temperature: the air isentropic index $k$ is 1.4 ; the air constant $R$ is 287 ; the gas isentropic index $k^{\prime}$ is 1.33 ; and the gas constant $R^{\prime}$ is 288
TABLE 1: Top-level parameters of a $230 \mathrm{~N}$ microturbojet engine [11].

\begin{tabular}{lc}
\hline Parameters & Value \\
\hline Total pressure ratio & 3.6 \\
Mass flow $(\mathrm{kg} / \mathrm{s})$ & 0.45 \\
Turbine inlet total temperature $(\mathrm{K})$ & 1023 \\
Rotating speed, $n(\mathrm{r} / \mathrm{min})$ & 100000 \\
\hline
\end{tabular}

TABle 2: Top-level parameter parameters of Jet-Cat P500-PRO$\mathrm{GH}$.

\begin{tabular}{lc}
\hline Parameters & Value \\
\hline Diameter $(\mathrm{mm})$ & 178.6 \\
Length $(\mathrm{mm})$ & 419 \\
Mass flow $(\mathrm{kg} / \mathrm{s})$ & 0.9 \\
Rotating speed $(\mathrm{r} / \mathrm{min})$ & 80000 \\
Total pressure ratio & 3.6 \\
Turbine outlet total temperature $(\mathrm{K})$ & 1013 \\
\hline
\end{tabular}

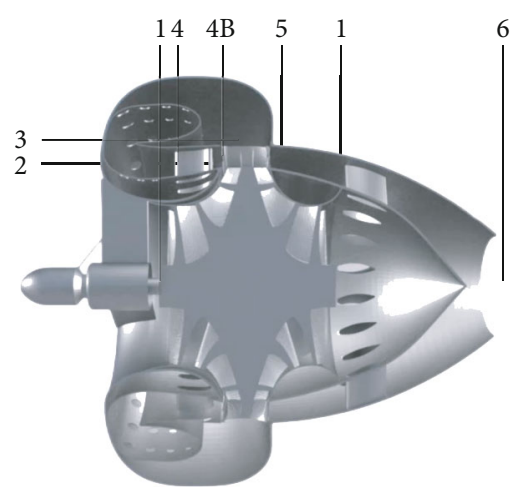

FIGURE 7: Cross-section annotations of the DCSI jet engine.

(4) To avoid introducing new problems, the hollow turbine guide is simplified to the turbine inlet absolute velocity boundary condition

(5) For analysis convenience, a convergent nozzle is used.

From these top-level parameters for the DSCI jet engine, the design parameters of each component can be calculated, such as the mass flow rate, total pressure ratio and efficiency. First, in accordance with the top-level parameters listed in Table 1, the aerodynamic parameters of the centrifugal compressor can be predictively calculated (considering the design size of a centrifugal compressor) [11]. Second, based on the top-level parameters listed in Table 2, the aerodynamic parameters of the turbine can be predictively calculated (considering double-sided centrifugal compressor power). Finally, with the help of these parameters, we can calculate the overall parameters of the DSCI and obtain its performance.

Regarding thermodynamic analysis, Figure 7 depicts the cross-section annotations of the DCSI jet engine. 


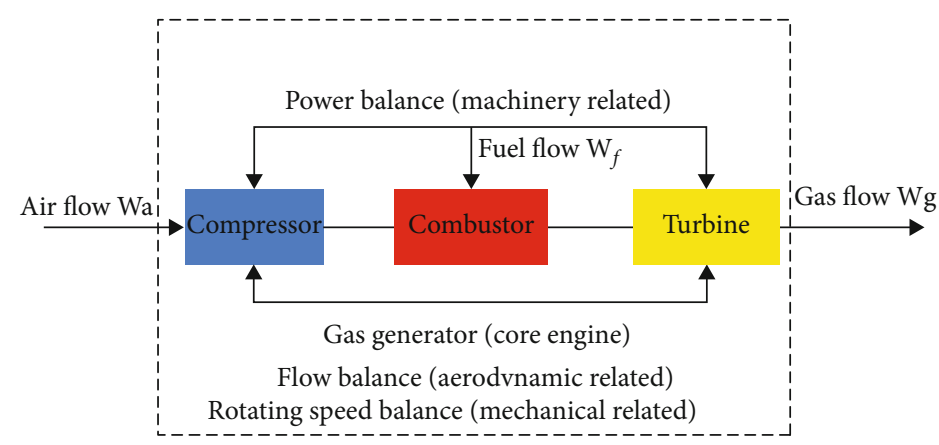

Figure 8: Flow chart of operating conditions of the gas generator components.

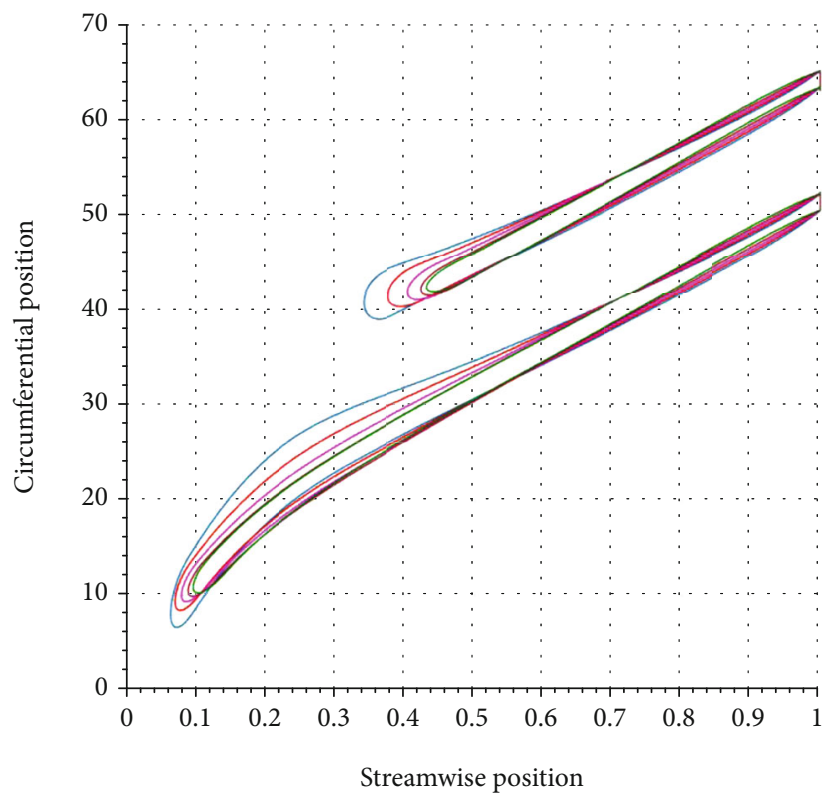

(a)

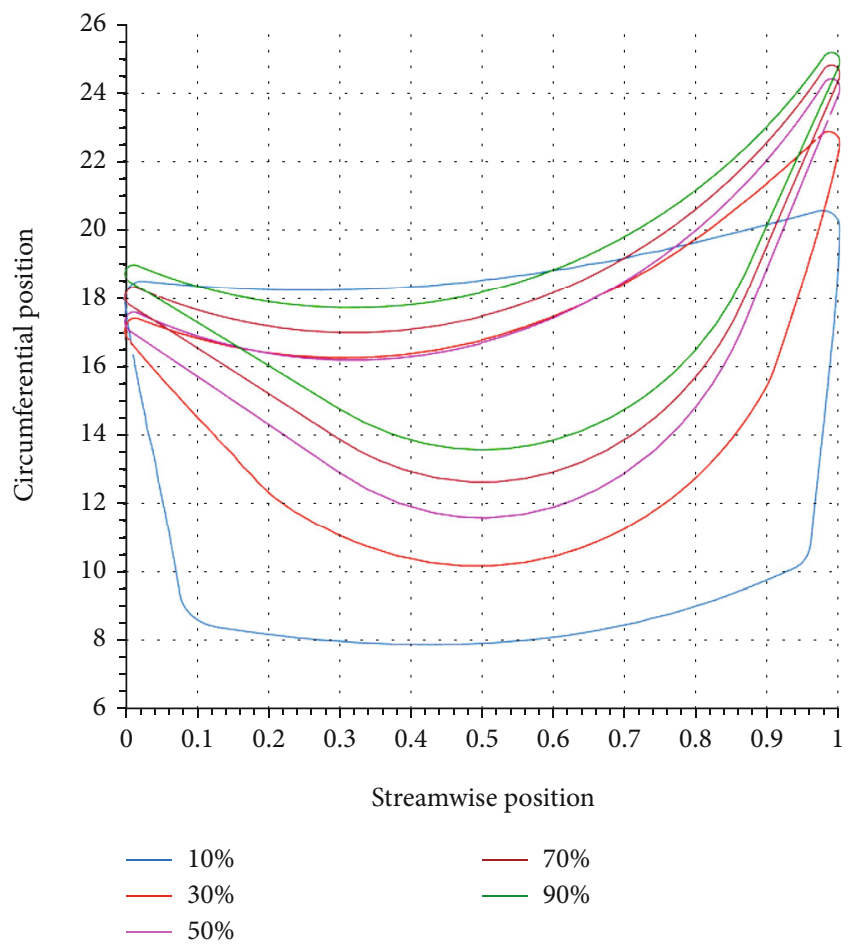

(b)

FIGURE 9: Section graphs of the DSCI (units: mm; 0\% represents the hub; 100\% indicates the tip). (a) Centrifugal compressor. (b) Hollow-tip turbine.

3.2. Preliminary Design Profiles of the DSCI Rotor. The design processes of the double-sided centrifugal compressor and the hollow-tip turbine are the same as those of other turbomachines, except the matching design process. These processes follow a preliminary design, throughflow design, 2D blading design, and 3D blading design [15]. Notably, the matching design process is necessary to meet the four matching balance conditions, as in the traditional gas generator shown in Figure 8 [16].

(1) Rotation speed balance: it is known that the turbine and the double-sided centrifugal compressor are radially connected, so the conditions are naturally met
(2) Flow balance: the sum of double-sided centrifugal compressor air flow and combustion chamber fuel flow is equal to turbine gas flow. The DSCI engine flow is continuous

(3) Power balance: the power required by the doublesided centrifugal compressor is equal to the turbine output power multiplied by the mechanical efficiency

(4) Pressure balance: the pressures between each section should be linked to each other in the DSCI engine.

Since the hollow-tip turbine is mounted at the tip of the double-sided centrifugal compressor, the two parts are attached to each other using a structural and 


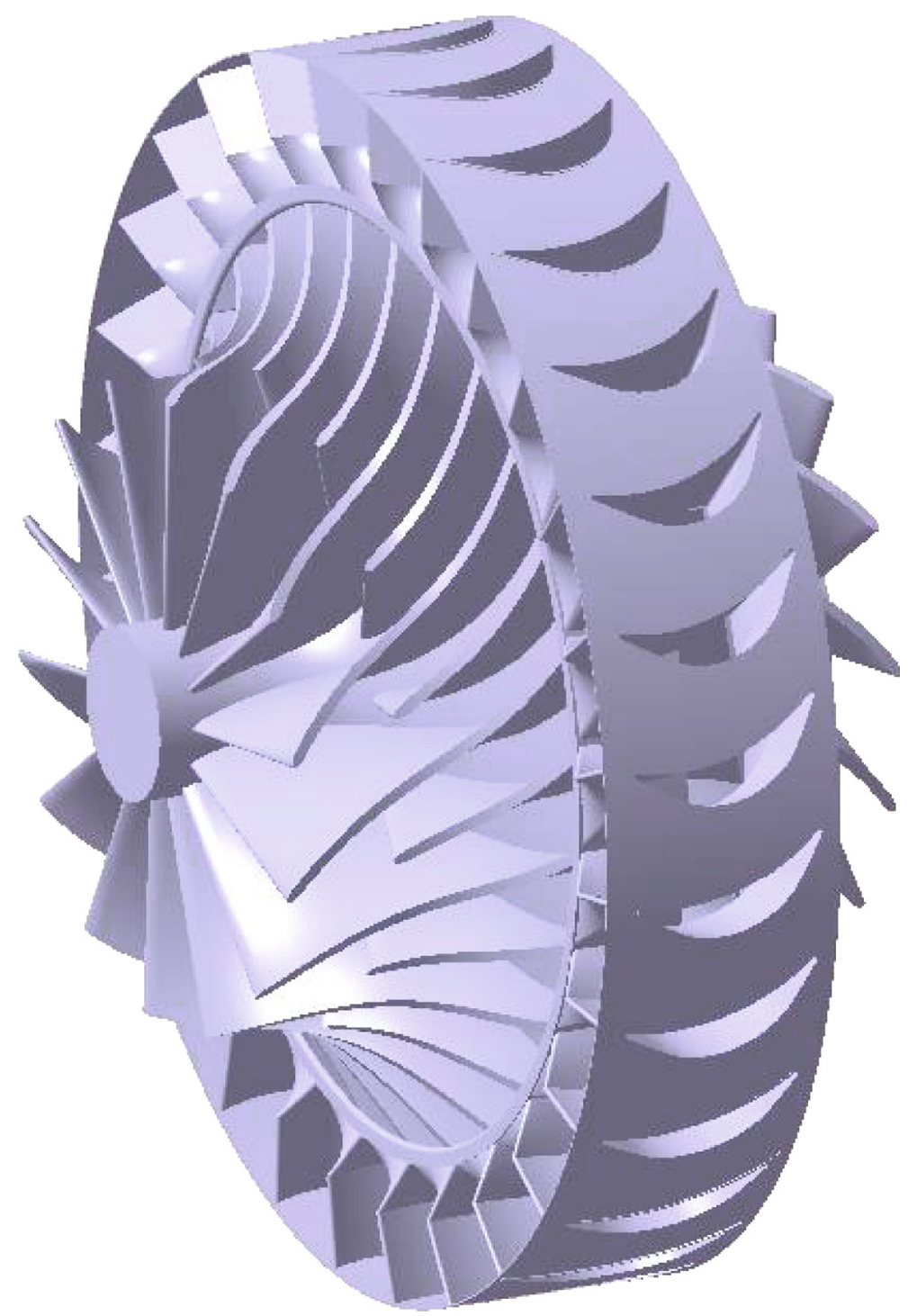

FIgURE 10: 3D model of the DSCI.

aerodynamic design. Several matching methods must be emphasized as follows:

(1) The number of blades of the double-sided centrifugal compressor and hollow-tip turbine must be equal (considering the feasibility of aerodynamics and rotor strength)

(2) As the diameter of the rotor increases at a given circumferential speed, the rotating speed should be reduced accordingly (the maximum circumferential speed of the rotor must be lower than $550 \mathrm{~m} / \mathrm{s}$ )

(3) The turbine has a skewed blade to adapt the diameter changes from hub to shroud (considering the turning loss in the high-pressure air inside the turbine)

(4) The profiles of the turbine must adopt the design rules for blade transition sections (considering that the exit section of the double-sided centrifugal compressor is rectangular).
TABLE 3: Design parameters of DSCI.

\begin{tabular}{lcc}
\hline $\begin{array}{l}\text { Design } \\
\text { parameters }\end{array}$ & $\begin{array}{c}\text { Double-sided centrifugal } \\
\text { compressor }\end{array}$ & $\begin{array}{c}\text { Values } \\
\text { Inside/outside the } \\
\text { turbine }\end{array}$ \\
\hline$\pi_{k}{ }^{*} / \pi_{T}{ }^{*}$ & 3.5 & $2.1 / 2.5$ \\
$\eta_{k}{ }^{*} / \eta_{T}{ }^{*}$ & 0.85 & $0.84 / 0.9$ \\
$n\left(10^{4} \mathrm{r} / \mathrm{min}\right)$ & 9 & 8 \\
$W_{a} / W_{g}(\mathrm{~kg} / \mathrm{s})$ & 0.9 & $0.84 / 0.85$ \\
$r_{1}(\mathrm{~mm})$ & 33.7 & 49.5 \\
$r_{2}(\mathrm{~mm})$ & 49.5 & 64 \\
$Z$ & 56 & 28 \\
$\varepsilon(\mathrm{mm})$ & 0.1 & 0 \\
$h(\mathrm{~mm})$ & -- & 14.5 \\
$\beta_{1 k}\left(^{\circ}\right)$ & -- & 60 \\
$\beta_{2 k}\left({ }^{\circ}\right)$ & 53 & 25 \\
$c_{\max }(\mathrm{mm})$ & -- & 24.6 \\
$\delta(\mathrm{mm})$ & 1 & 0.3 \\
\hline
\end{tabular}




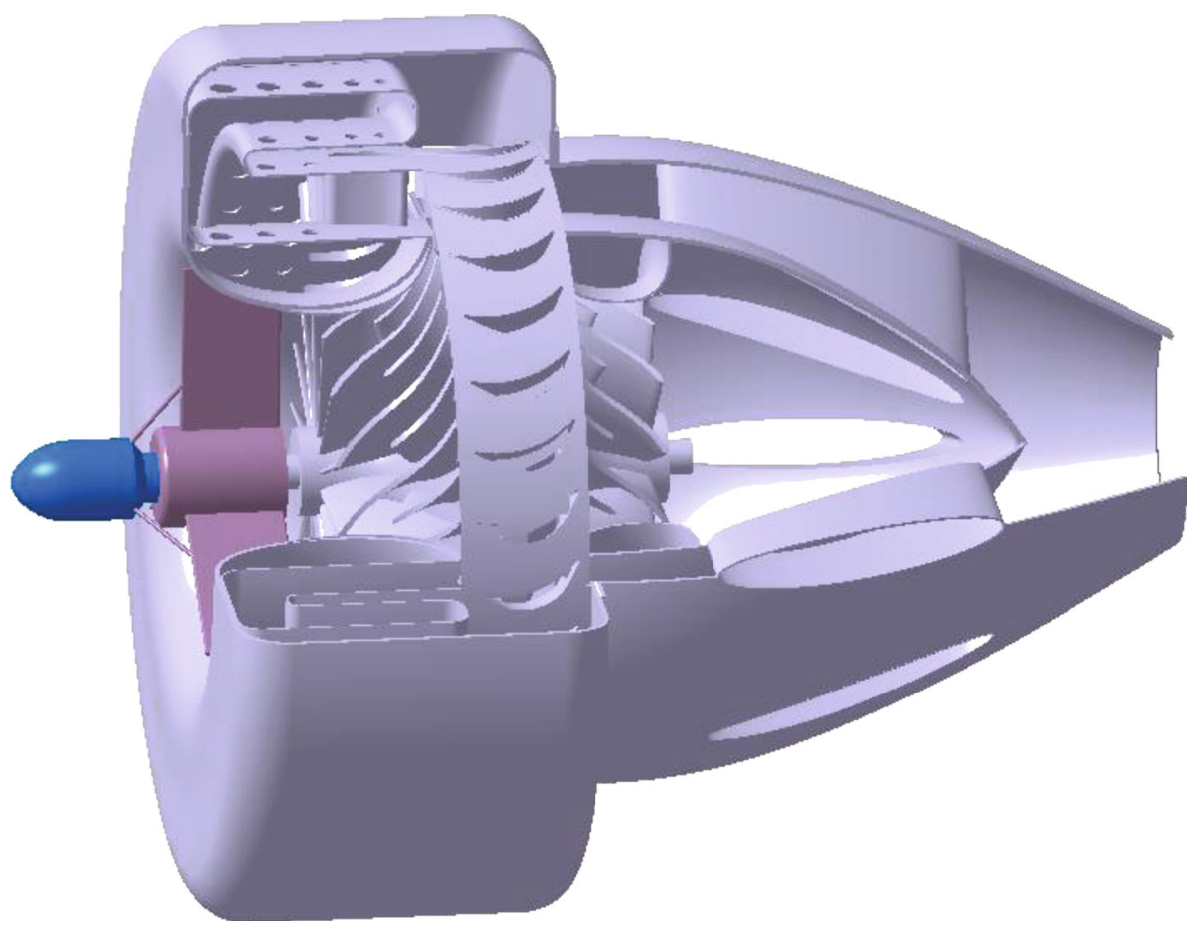

FIgURE 11: The structure of the DSCI jet engine.

Profiles of the DSCI are depicted in Figure 9. In the preliminary design, since the turbine needs to meet the high load, the shape of the turbine must be designed as an impact turbine with a counterforce. On the other hand, the turbine is different from the conventional axial turbine in the transition section (defined from the hub to the 0.3 span). The inside profiles of the turbine adopt a similar tubular diffuser structure [17]. All the blades are stacked near the center of gravity, and the 3D structure of the double-sided centrifugal compressor and hollow-tip turbine can be seen in Figure 10 .

According to the above top-level parameters and assumptions, using the design parameters of the DSCI listed in Table 3, the design performance of the DSCI jet engine can be calculated.

3.3. Preliminary Design of the DSCI Jet Engine. To facilitate the assessment of the structural weight with the DSCI jet engine, the engine model is preliminarily designed, as shown in Figure 11. By evaluating the weight of each part in the engine, the DSCI jet engine weight can be predicted, as shown in Table 4 .

The preliminary design of the DSCI rotor and its supporting DSCI jet engine was carried out in the previous section, but the feasibility of DSCI and the performance parameters of the DSCI jet engine need to be verified. These topics will be discussed subsequently.

\section{Thermodynamic Calculation and Analysis of the DSCI Jet Engine under Design Parameters}

Currently, computational fluid dynamics (CFD) plays an important role in the aerodynamic design of turbomachines.
TABLE 4: Weight parameters of DSCI jet engine.

\begin{tabular}{lcc}
\hline Part name & Weight $G(\mathrm{~kg})$ & Material \\
\hline DSCI & 1.563 & GH93 \\
Main shaft & 0.027 & GH93 \\
Casing and combustor & 1.081 & NAS800 \\
Nozzle and rectifier cone & 0.625 & NAS800 \\
Starting dynamo & 0.787 & - \\
Bearing & 0.01 & Babbitt metal \\
Fuel injection ring & 0.023 & NAS800 \\
Oil pump & 0.065 & - \\
Oil filter & 0.02 & - \\
Flame ignitor & 0.01 & - \\
Inlet guide vane & 0.028 & Steel \\
DSCI jet engine & 4.239 & - \\
\hline
\end{tabular}

To estimate the preliminary aerodynamic design of the DSCI, the DSCI rotor, an innovative and uncertain component, is certified through CFD and theoretical analysis. In addition, the performance parameters of the DSCI jet engine are also evaluated and compared with the JetCat P500-PRO-GH.

4.1. CFD Method. The ANSYS CFX commercial CFD software, including the meshing module ANSYS meshing and computing module CFX-TASCflow, was used for numerical simulation.

The fluid domains of the DSCI inside, the turbine outside, and the solid domain of the turbine are meshed by ANSYS meshing. The turbine inside contains 1.44 million 


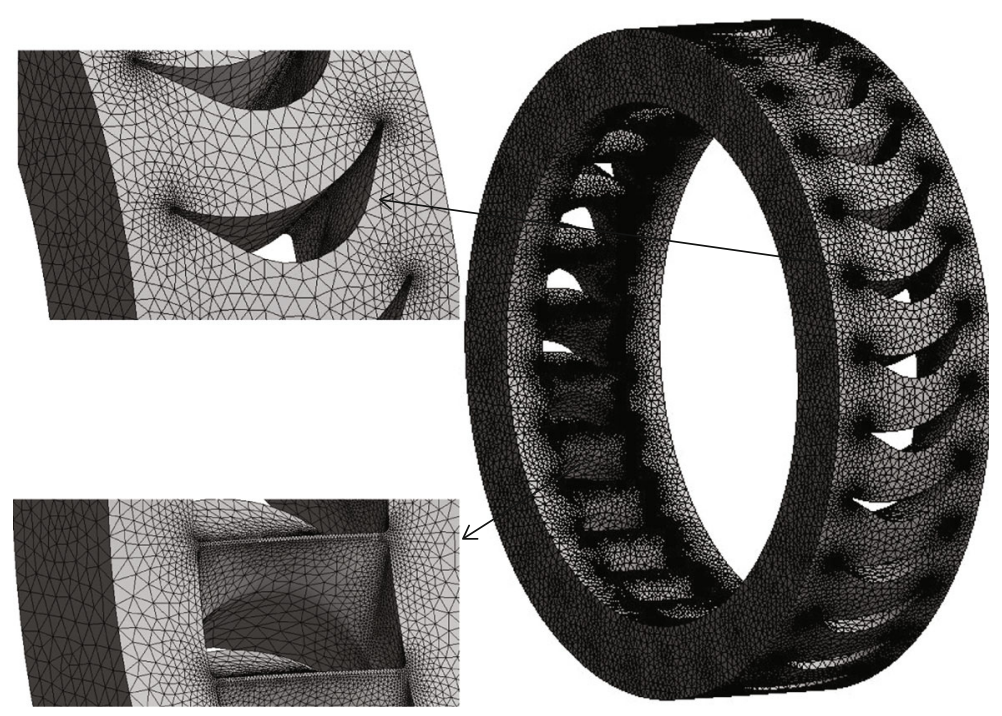

(a)

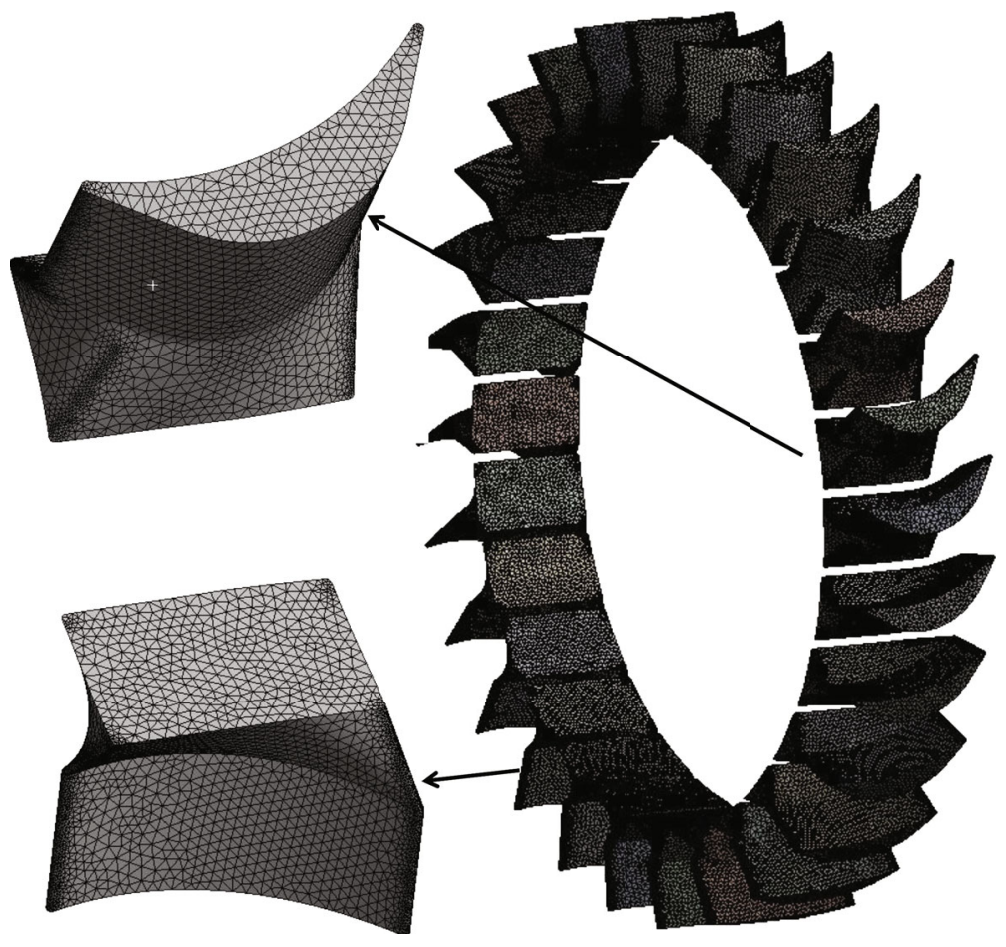

(b)

Figure 12: Continued. 

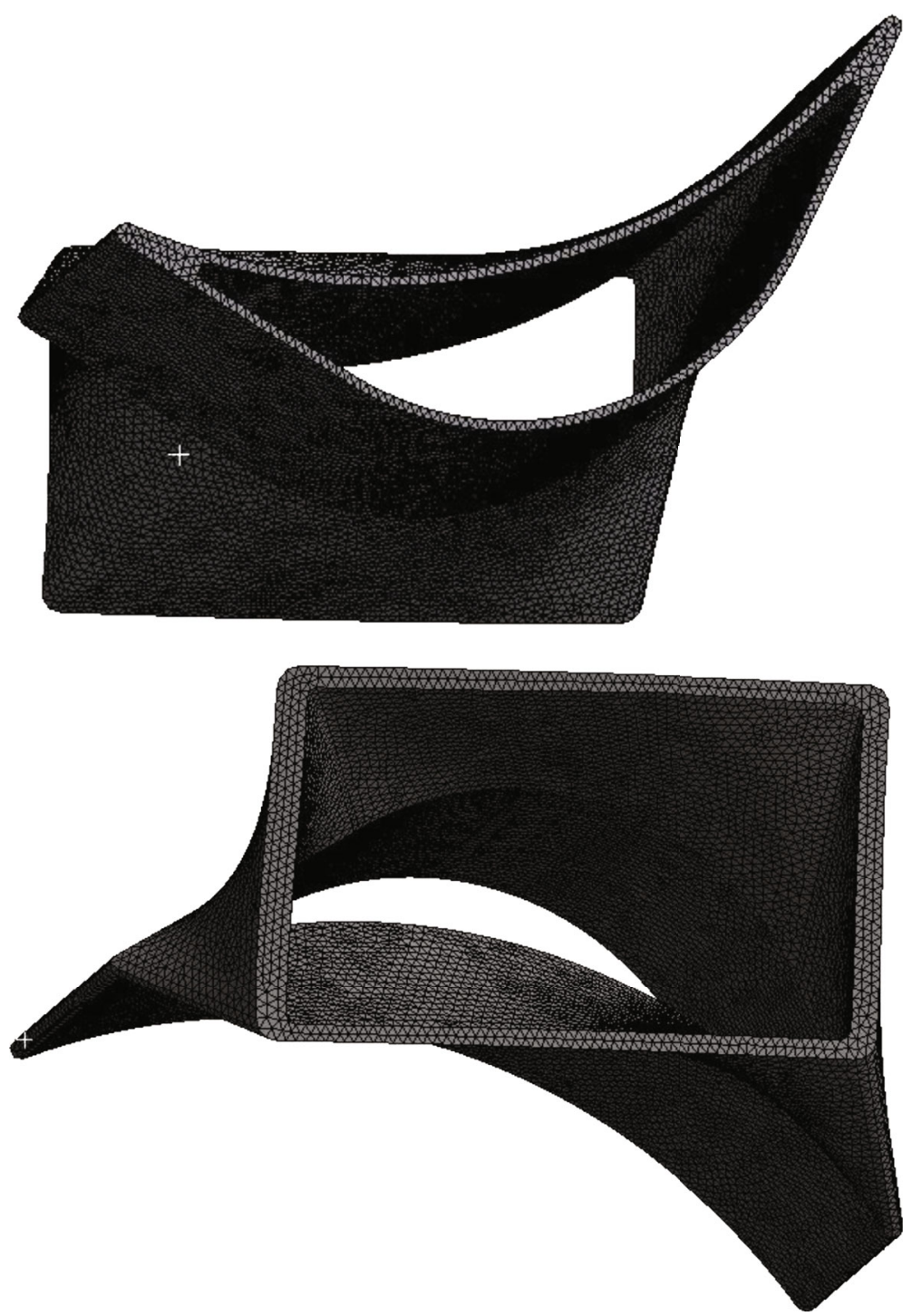

(c)

Figure 12: Continued. 


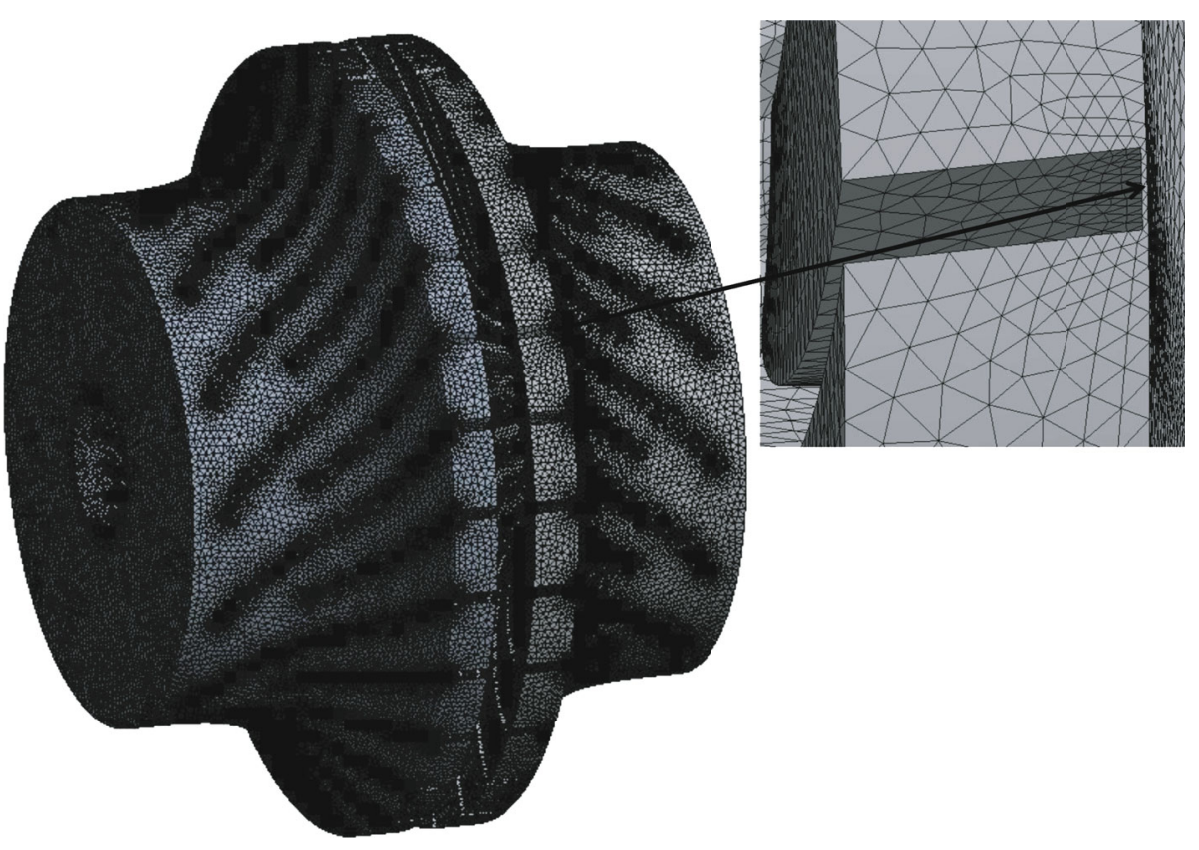

(d)

FIGURE 12: Each domain grid of the DSCI. (a) Outside the turbine fluid domain. (b) Inside the turbine fluid domain grids. (c) Turbine solid domain grids. (d) Double-sided centrifugal compressor grids.

TABLE 5: The fluid domain boundary conditions of the DSCI inside and outside.

\begin{tabular}{lcc}
\hline Boundary conditions & & Fluid domains \\
\hline Inlet & Fluid domain of DSCI inside & Fluid domain of DSCI outside \\
Outlet & Total temperature, Total pressure & Total temperature, velocity components \\
Turbulence & Static pressure & Static pressure \\
\hline
\end{tabular}

fluid domain grids, the turbine outside contains 3.09 million fluid domain grids, the turbine contains 2.75 million solid domain grids, and the double-sided centrifugal compressor contains 3.14 million fluid domain grids, as shown in Figure 12. Through grid quality inspection, it is found that the maximum skewness is lower than 0.95 and that the average orthogonal quality is higher than 0.75 , which indicates that these grids can be used for numerical simulation. Theoretically, the accuracy of a tetrahedral mesh (considering first-order elements) may be slightly lower when compared with a hexahedral mesh, but its self-adaptive mesh technology can be applied to any complex geometry and provide for mesh refinement of high stress gradient parts. The advantages of automatic grid encryption save cost and time.

A steady-state model and high-resolution discretization scheme were used for simulations. The discretized form of Reynolds Averaged Navier-Stokes (RANS) equations was iteratively solved for the DSCI. Since the SST model is used in turbomachinery, in most cases, it requires $Y^{+}$to be very small $\left(Y^{+}<2\right)$. Therefore, the layer mesh quality requirement of the SST model is higher than that of the $k$-epsilon model. Reference [18] demonstrated that when the computational grid number is very large, the $k$-epsilon
Table 6: Properties of the GH93 material.

\begin{tabular}{lccc}
\hline Static temperature, $\mathrm{K}$ & $\begin{array}{c}\lambda \\
\left(\mathrm{W} \mathrm{m}^{-1} \mathrm{~K}^{-1}\right)\end{array}$ & $\begin{array}{c}C_{p} \\
\left(\mathrm{~J} \mathrm{~kg}^{-1} \mathrm{~K}^{-1}\right)\end{array}$ & $\begin{array}{c}\text { Density } \\
\left(\mathrm{g} \mathrm{cm}^{-3}\right)\end{array}$ \\
\hline 373 & 12.77 & 467 & \\
473 & 14.44 & 494 & \\
573 & 15.99 & 520 & \\
673 & 17.54 & 547 & \\
773 & 18.97 & 572 & \\
873 & 20.64 & 600 & 8.19 \\
973 & 22.32 & 626 & \\
1073 & 23.99 & 652 & \\
1173 & 25.83 & 679 & \\
1273 & 27.88 & 706 & \\
1373 & 30.06 & 733 & \\
1473 & 32.47 & 760 & \\
\hline
\end{tabular}

model is used as the turbulence model in simulating turbomachinery in order to relax the demand on computer memory and raise the efficiency. The calculated residuals converge below $10^{-5}$. 

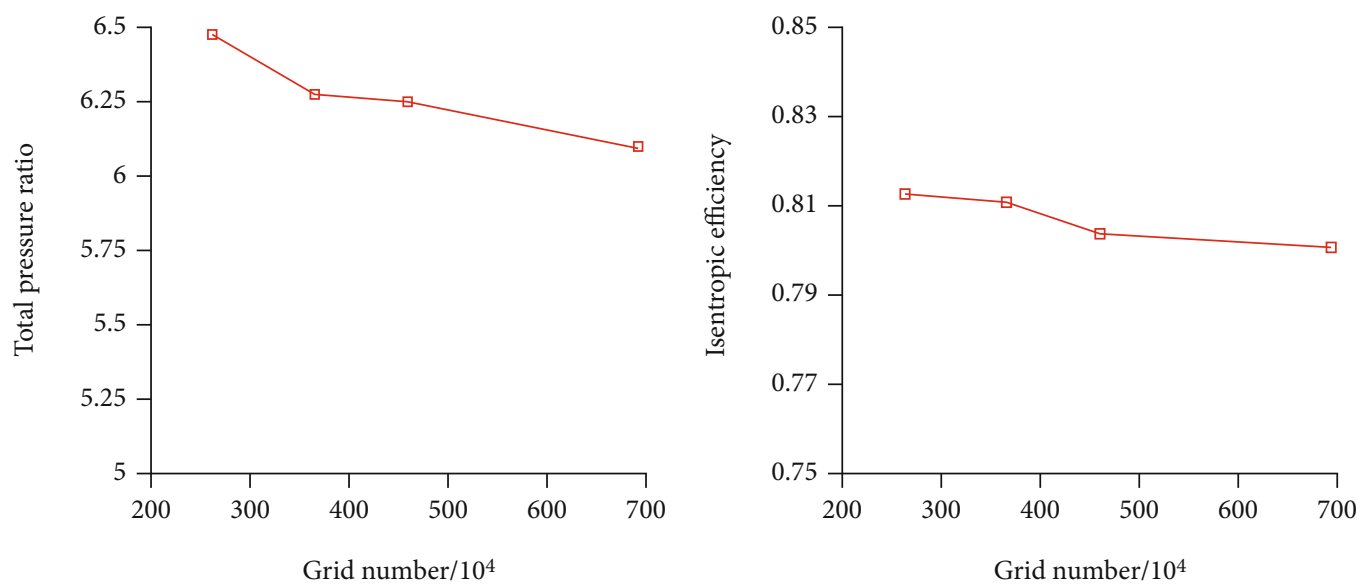

(a)
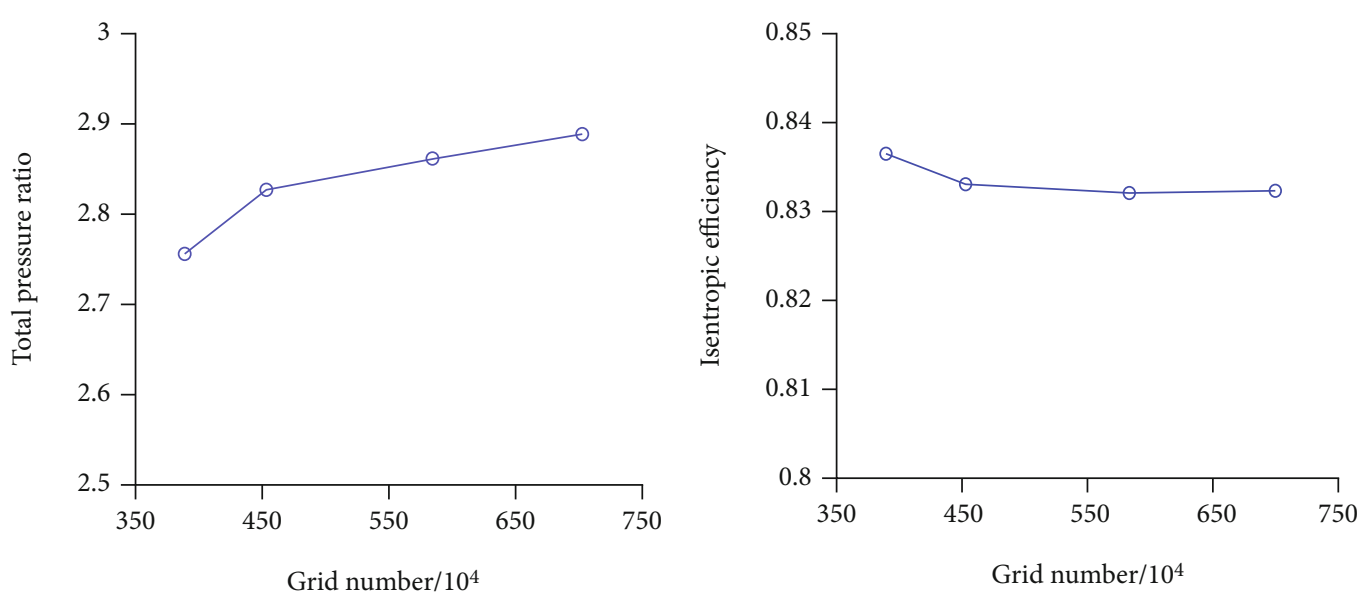

(b)

FIGURE 13: Grid independence verification of turbomachines. (a) DSCI. (b) Turbine.

The boundary conditions are listed in Table 5 and are as follows: the nonsliding solid wall treatment method is used, except the fluid-solid interface is set to exchange heat [19, 20]; the DSCI surfaces are set rotating, except that the shroud is a static boundary; the double-sided centrifugal compressor inlet total pressure $P_{\mathrm{t} 1}$ is $101325 \mathrm{~Pa}$; the double-sided centrifugal compressor inlet total temperature $T_{\mathrm{t} 1}$ is $288 \mathrm{~K}$; the turbine inside outlet static pressure $P_{3}$ is $212783 \mathrm{~Pa}$; the turbine outside inlet total temperature $T_{\mathrm{t}}$ is $1150 \mathrm{~K}$; the turbine outside inlet axial velocity component $C_{\mathrm{a} 4 \mathrm{~B}}$ is $149.5 \mathrm{~m} / \mathrm{s}$; the turbine outside inlet circumferential velocity component $C_{\tau 4 \mathrm{~B}}$ is $590 \mathrm{~m} / \mathrm{s}$; and the turbine outside outlet static pressure $P_{5}$ is $202650 \mathrm{~Pa}$.

Based on the property parameters of the GH93 material listed in Table 6 [21], the missing data can be calculated using the least squares method in MATLAB.

The grid independency is examined via increasing the node number until the evaluated design point performance of turbomachines is essentially no longer changed. As Figure 13 shows, the calculated efficiency and total pressure ratio of DSCI and turbine have converged with 4.58 million and 5.84 million grids, respectively, which are considered to grid size effect independent. Otherwise mentioned, the com- putation model grid number in the later calculations is about 10.42 million, as shown in Figure 14 [22-25].

\subsection{CFD Results and Discussions}

4.2.1. Compression Process. First, Figure 15 displays several contours of the double-sided centrifugal compressor under the design point. From left to right, there are the auxiliary impeller and the main impeller. In accordance with the centrifugal pressurization principle, the static pressure gradually increases from the inlet to the outlet of the meridian channel in the double-sided centrifugal compressor, as shown in Figures 15(a) and 15(b).

Second, since the inside shape of turbine is asymmetrical along the mirror plane in Figures 15(c) and 15(d), the flow field of main impeller is different from that of auxiliary impeller.

Finally, because the leakage vortex caused by the angleof-attack induces tip clearance flow and blade radial shear flow, there are more high entropy values (low-momentum air) in the tip area of the double-sided centrifugal compressor. When the leakage vortex is extreme, it spreads to the main flow area of the impeller, leading to impeller compression difficulties and reduced efficiency. 


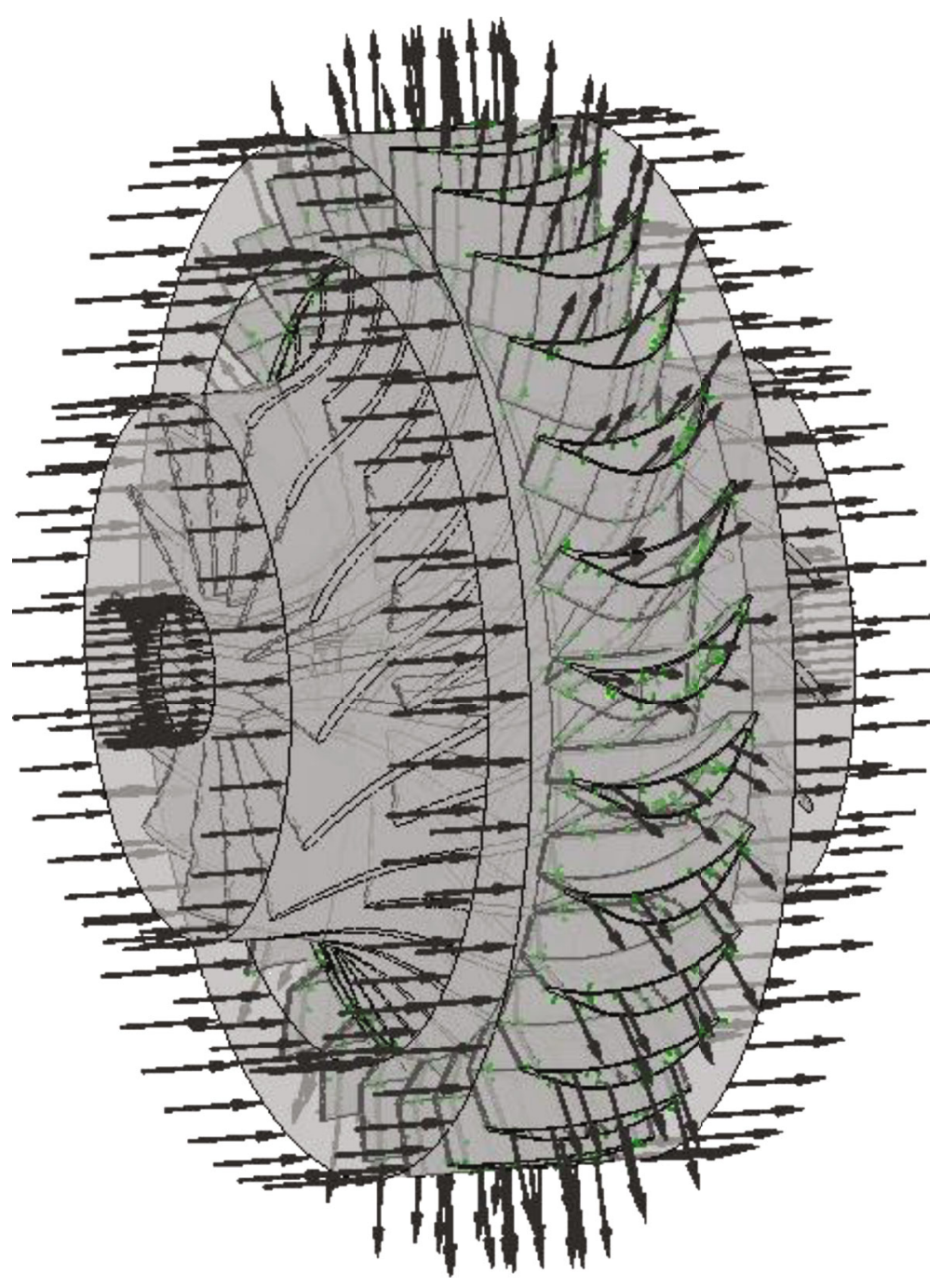

FIgUre 14: Calculation model of the DSCI.

Figures 16-18 show the blade-to-blade planes of the double-sided centrifugal compressor under the design point. From left to right, there are the main impeller and the auxiliary impeller. As the blade height increases, the aerodynamic load of the blade increases, as shown in Figure 16. Due to the influence of the high adverse pressure gradient at the tip of the centrifugal compressor, the tip leakage vortex is easily induced, and in severe cases, the impeller will stall in advance. Figures 17 and 18 further confirm the above analysis. In Figure 17, it can be observed that the main impeller has produced some stall clusters at the 0.9 span.

In summary, due to the influence of the downstream inside turbine, in the main and auxiliary impellers of the same size, the main impeller is closer to the surge point, so the compression capacity of the main impeller is better, but the tip flow loss is greater.

The double-sided centrifugal compressor is defined as the first stage compression section in Figure 15.

First, when these two flows exit the double-sided centrifugal compressor, they will mix with each other, thereby resulting in the loss of mixture. Because the total pressure ratio and isentropic efficiency of the main and auxiliary impellers are different from each other, the compression performance of the double-sided centrifugal compressor cannot be estimated. Since these two flows exit the doublesided centrifugal compressor and enter the inside of the turbine at the same time, the inlet section of the turbine inside can be used as the calculated outlet section of the double-sided centrifugal compressor. The first stage compression section total pressure ratio $\pi_{c 1}{ }^{*}$ is 2.901 , and its isentropic efficiency $\eta_{c 1}{ }^{*}$ is 0.8538 as calculated by the numerical simulation results and the following formulas:

$$
\begin{aligned}
& \pi_{c 1}{ }^{*}=\frac{P_{t 2}}{P_{t 1}}, \\
& \eta_{c 1}^{*}=\frac{\pi_{c 1}{ }^{(k-1) / k}-1}{\left(T_{t 2} / T_{t 1}\right)-1},
\end{aligned}
$$

where $P_{t 1}$ refers to the double-sided centrifugal compressor inlet total pressure, $T_{t 1}$ refers to the double-sided centrifugal compressor inlet total temperature, $P_{t 2}$ refers to the turbine 


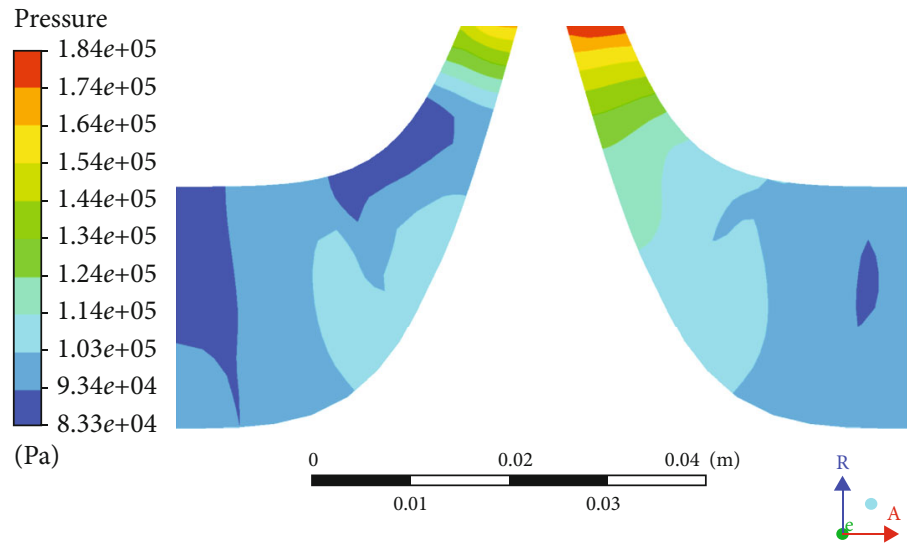

(a)

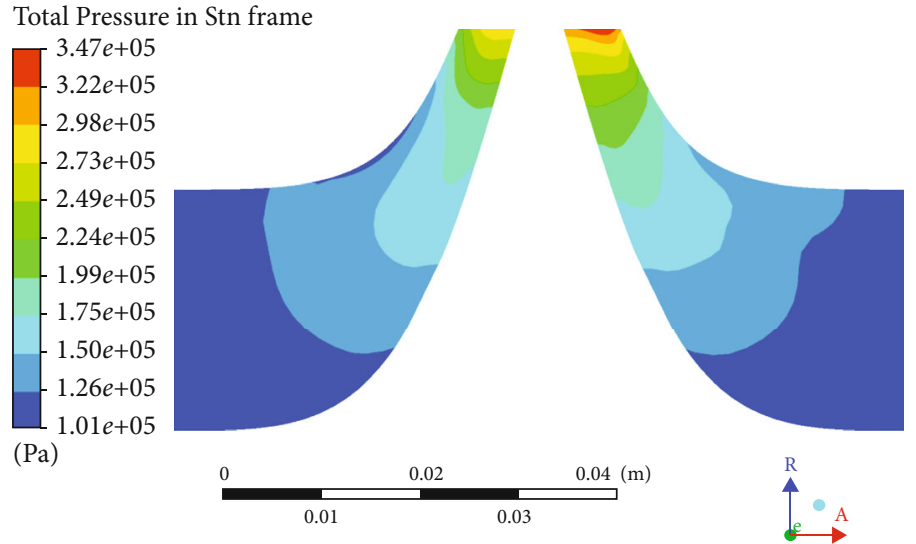

(b)

Mach number

0.98
0.90
-0.82
0.75
0.67
0.59
0.51
-0.43
0.36
0.28
0.20

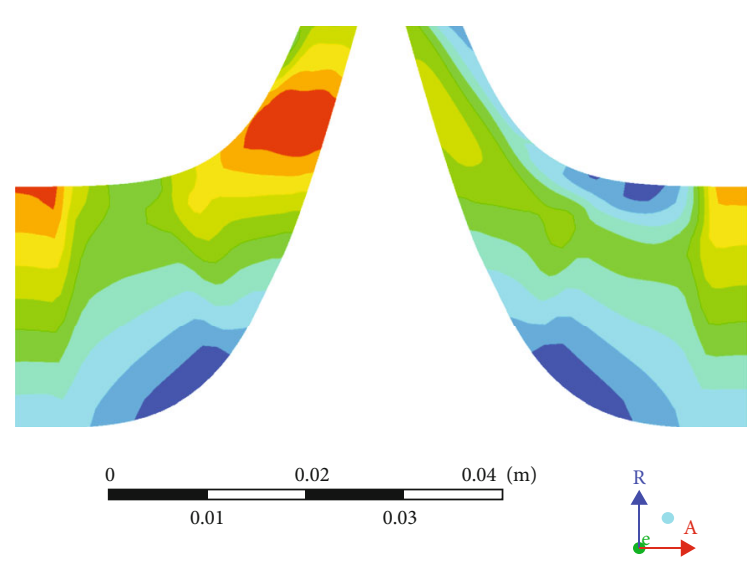

(c)

Figure 15: Continued. 


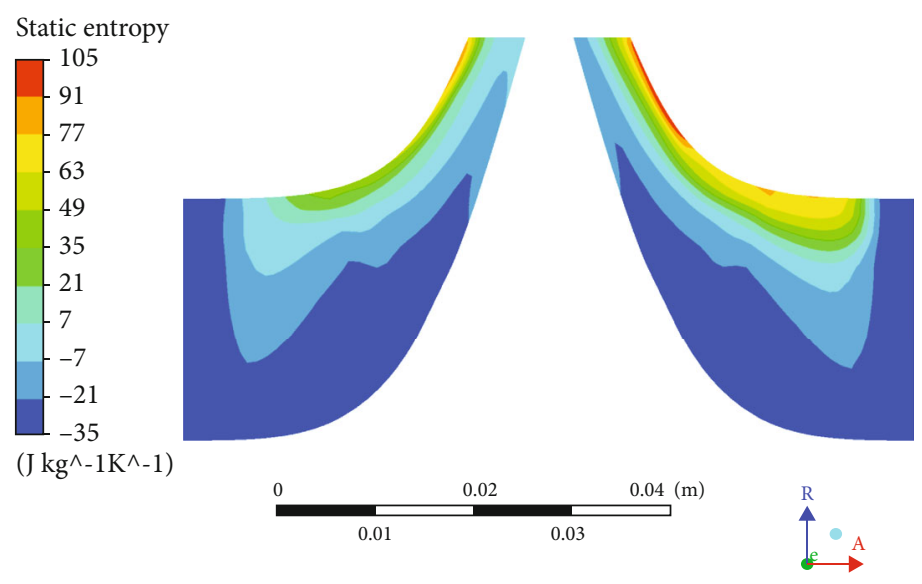

(d)

FIGURE 15: Several contours of the double-sided centrifugal compressor. (a) Static pressure. (b) Total pressure in Stn frame. (c) Relative Mach number. (d) Static entropy.

inside inlet total pressure, and $T_{t 2}$ refers to the turbine inside inlet total pressure.

Second, since the turbine is also rotating at the same speed as the double-sided centrifugal compressor, the highpressure air is also compressed inside the turbine. For ease of expression, this process is defined as the internal channel diffusion of the hollow-tip turbine. The total pressure ratio of this process $\pi_{c 2}{ }^{*}$ is 2.053 , and the isentropic efficiency $\eta_{c 2}{ }^{*}$ is 0.7081 as calculated by CFD.

Finally, the OPR of the compression process $\pi_{k}{ }^{*}$ is 5.957, and the overall isentropic efficiency $\eta_{k}{ }^{*}$ is 0.7617 by formulas (3) and (4):

$$
\begin{aligned}
& \pi_{k}{ }^{*}=\pi_{c 1}{ }^{*} \pi_{c 2}{ }^{*}, \\
& \eta_{k}{ }^{*}=\frac{\pi_{k}{ }^{*(k-1) / k}-1}{\left(T_{t 3} / T_{t 1}\right)-1},
\end{aligned}
$$

where $T_{t 3}$ refers to the outlet total temperature inside the turbine and $T_{t 1}$ refers to the total temperature of the double-sided centrifugal compressor inlet.

The isentropic efficiency of any single-stage compressor is generally higher than that of a multistage compressor. In this section, since there is convection heat transfer inside and outside the turbine, $\eta_{c 2}{ }^{*}$ is far below $\eta_{k}{ }^{*}$. Specifically, the high-temperature gas outside the turbine transfers heat to the high-pressure air that is being compressed in the turbine, resulting in $\eta_{c 2}{ }^{*}$ being lower than the overall isentropic efficiency $\eta_{k}{ }^{*}$.

Based on the above analysis, Table 7 displays the crosssectional parameters of the compression process. The compressor power $W_{k}$ is $252.6 \mathrm{~kJ} / \mathrm{kg}$ and is calculated by

$$
W_{k}=\frac{k}{k-1} R T_{t 1}\left(\pi_{k}^{* k-1 / k}-1\right) / \eta_{k}^{*}
$$

4.2.2. Combustion Process. To avoid introducing new problems and a large number of calculations, the traditional reverse-flow annular combustor was adopted. The fuel-air ratio $f_{b}$ is 0.017 at $T_{t 3}$ and $T_{t 4}$, and the combustor heat balance equation can be written as

$$
f_{b}=\frac{i_{t 4}-i_{t 3}}{\xi_{b} H_{f}-I_{4}+i_{t 3}}
$$

where $i_{t 4}, i_{t 3}$, and $I_{4}$ can all be determined with a look-up table and $H_{f}$ is $42900 \mathrm{~kJ} / \mathrm{kg}$ [26].

The fuel mass flow $m_{f}$ is $0.014 \mathrm{~kg} / \mathrm{s}$, and the gas mass flow $W_{g}$ is $0.854 \mathrm{~kg} / \mathrm{s}$ as calculated by the formulas for the fuel-air ratio and gas mass flow:

$$
\begin{gathered}
m_{f}=W_{a} f_{b}, \\
W_{g}=W_{a}+m_{f} .
\end{gathered}
$$

Notably, for the total pressure loss in the combustor, the total pressure recovery coefficient $\sigma_{b}$ is empirically given as 0.98 . The combustor outlet total pressure $P_{t 4}$ is $591498 \mathrm{~Pa}$, as calculated by

$$
P_{t 4}=\sigma_{b} P_{t 3}
$$

4.2.3. Expansion Process (outside the Turbine). After analyzing the combustion process, the expansion process needs to be further studied. The profile design of the hollow-tip turbine is different from that of a traditional turbine, especially the transition section. Therefore, it is necessary to study the flow field and characteristics of the turbine.

Figure 19 illustrates the static pressure contours of the turbine. High-temperature gas pressure is released in the turbine rotor channel and then drives rotor acceleration. Because the turbine root is part of the transition section and the circumferential velocity is small, the pressure drop ratio is small at the blade root, regardless of differing blade height. 


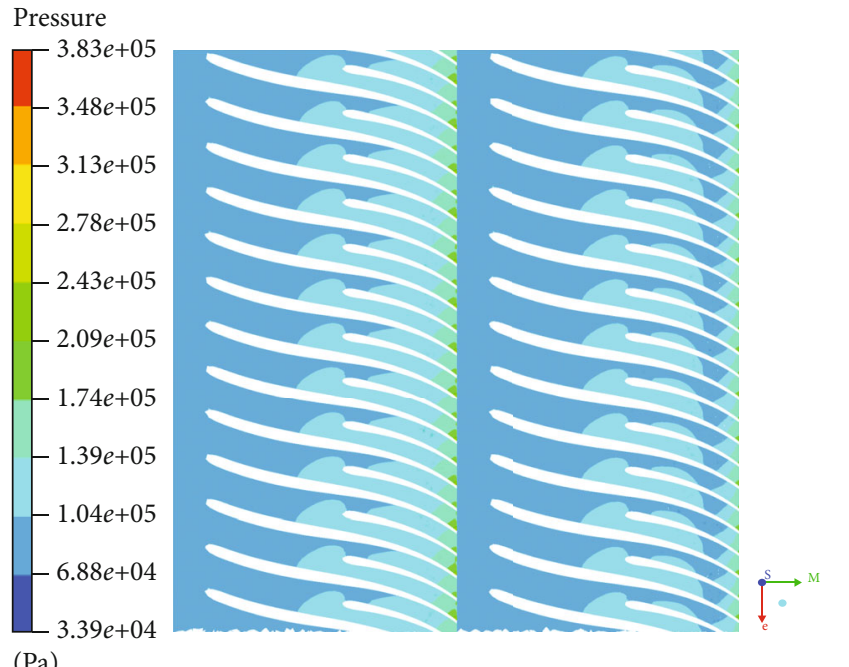

(a)

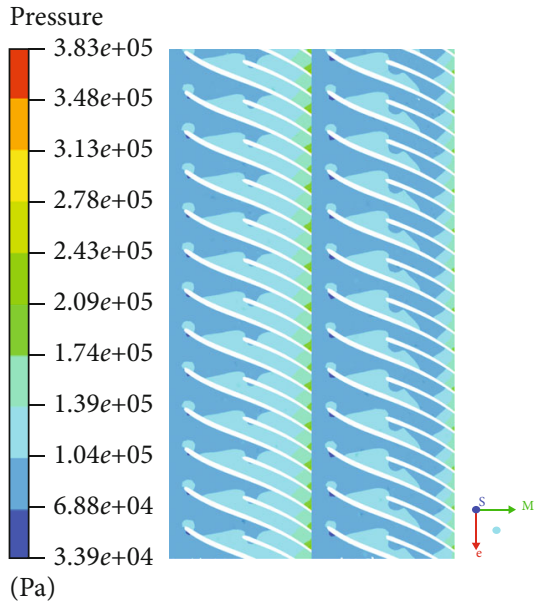

(b)

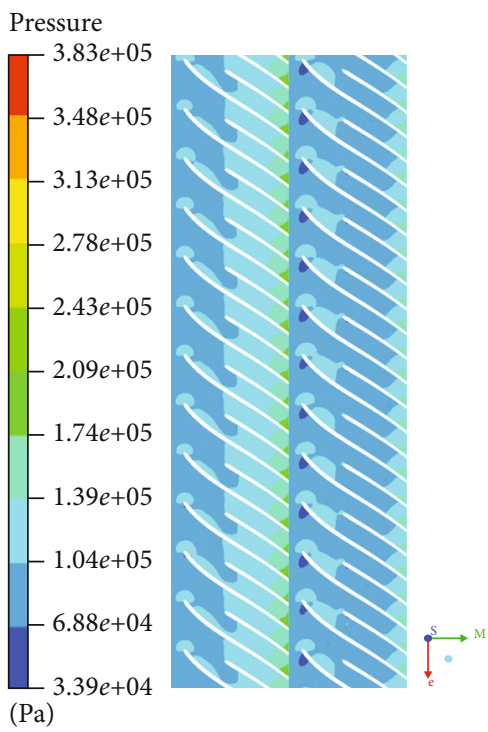

(c)

FIGURE 16: Blade-to-blade plane of double-sided centrifugal compressor for static pressure. (a) 0.1 span. (b) 0.5 span. (c) 0.9 span.

Figure 20 shows the relative Mach number contours of the turbine. The high-temperature gas accelerates and depressurizes in the turbine rotor channel and then drives the DSCI rotor to rotate. The maximum relative Mach number appears at the throat of the turbine, which corresponds to the low-pressure area in Figure 19, indicating that the turbine throat outlet section is supersonic and generates a set of shock waves. This set of expansion shock waves hit the trailing edge of the suction surface of the adjacent turbine blades and interfere with the boundary layer, causing more serious loss of the boundary layer. As the height of the turbine blade increases, this phenomenon becomes more prominent.

Figure 21 shows the static entropy contours of the turbine channel. The change in the angle-of-attack causes the phenomenon of separated flow or even back flow in the transition section, namely, the loss of turbine profiles. Comparing the different blade heights, the high entropy value of the turbine is mainly distributed near the trailing edge of the suction surface. This indicates that the supersonic velocity of the throat section of the turbine produces a set of expansion shock waves. These waves hit the trailing edge of the suction surface of the adjacent turbine and interfere with the boundary layer, causing a serious loss of the boundary layer.

Based on the above, the loss of turbine profiles and the shock wave boundary layer interference loss are the main reasons for the decrease of the turbine efficiency.

Figure 22 shows the relative velocity vector contours of the turbine. There is a certain deflection angle between the inlet relative flow angle and the inlet geometry angle at the turbine root, namely, a large positive angle-of-attack. This indicates that there is flow separation at the leading edge of the suction surface. However, as the height of the turbine increases, the tangential velocity also increases, and the 


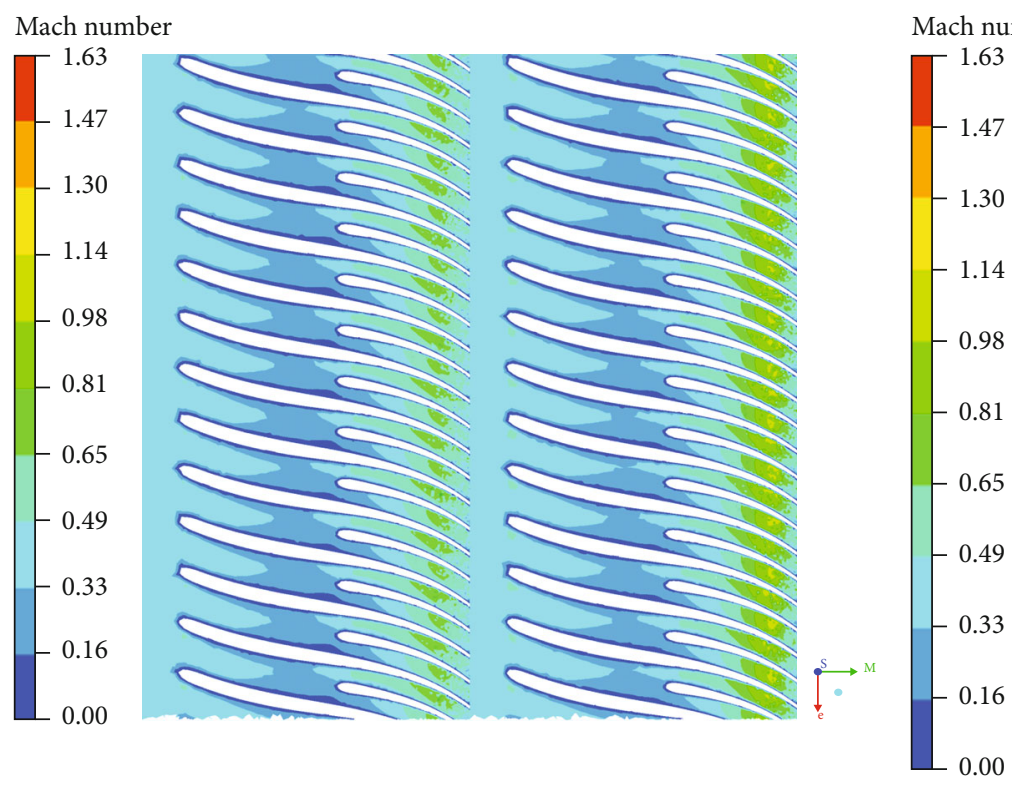

(a)

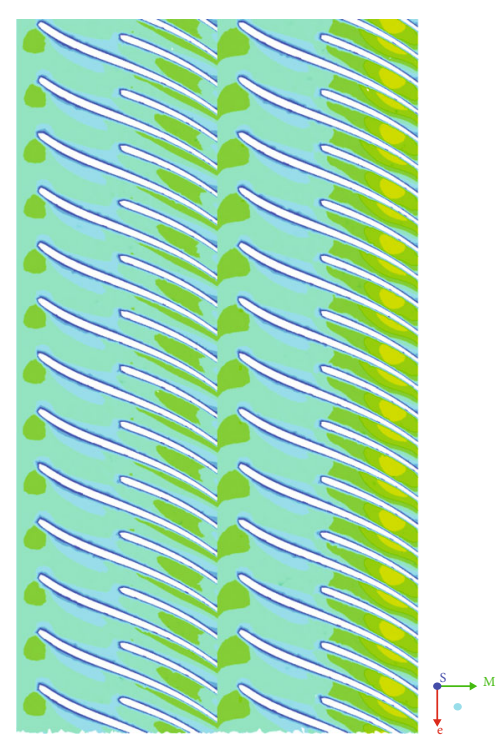

(b)

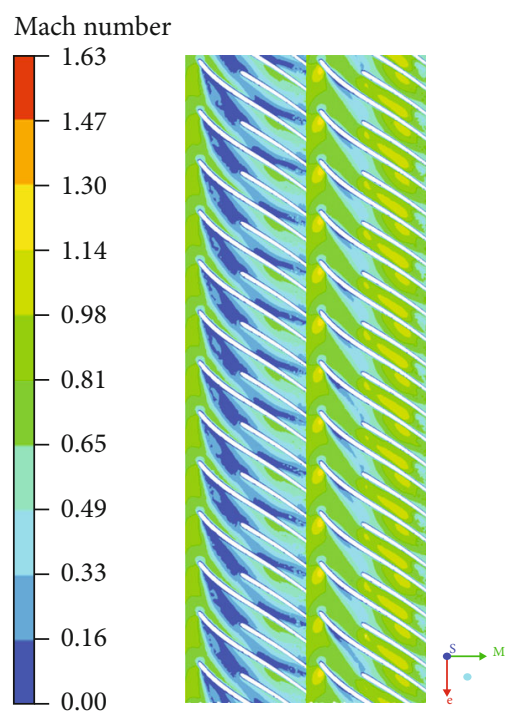

(c)

FIGURE 17: Blade-to-blade planes of double-sided centrifugal compressor for relative Mach number. (a) 0.1 span. (b) 0.5 span. (c) 0.9 span.

corresponding relative flow angle also increases. The reduction of the positive angle-of-attack causes the flow separation at the leading edge to weaken. For this kind of problem, further optimization is possible by twisting the turbine root in the later stage.

Figure 23 shows the static pressure distribution of the turbine. The static pressure load difference between the pressure surface and the suction surface is small at the 0.1 span of the turbine, which further illustrates that the expansion of the turbine transition section is a poor function. As the leaf height increases, the expansion function is improved. At the turbine leading edge, the static pressure load on the suction side is greater than that on the pressure side, indicating that it is not appropriate to set a sharp front edge contour shape. In the later stage, general antiforce axial turbines can be considered.

Figure 24(a) displays the static temperature contours of the DSCI wall. Although the cooling coefficient inside the turbine is close to 1 and the high-pressure gas has a good cooling effect on the turbine wall, there is a local high temperature at the trailing edge of the blade and the hub of the double-sided centrifugal compressor due to the heat conduction in the solid. Thus, the high local temperature may slightly affect the compression and structural strength of the DSCI, which needs to be further researched.

Figure 24(b) displays the static temperature contour outside the turbine wall. The maximum higher temperature is mainly distributed at the tip of the leading and trailing edges, 


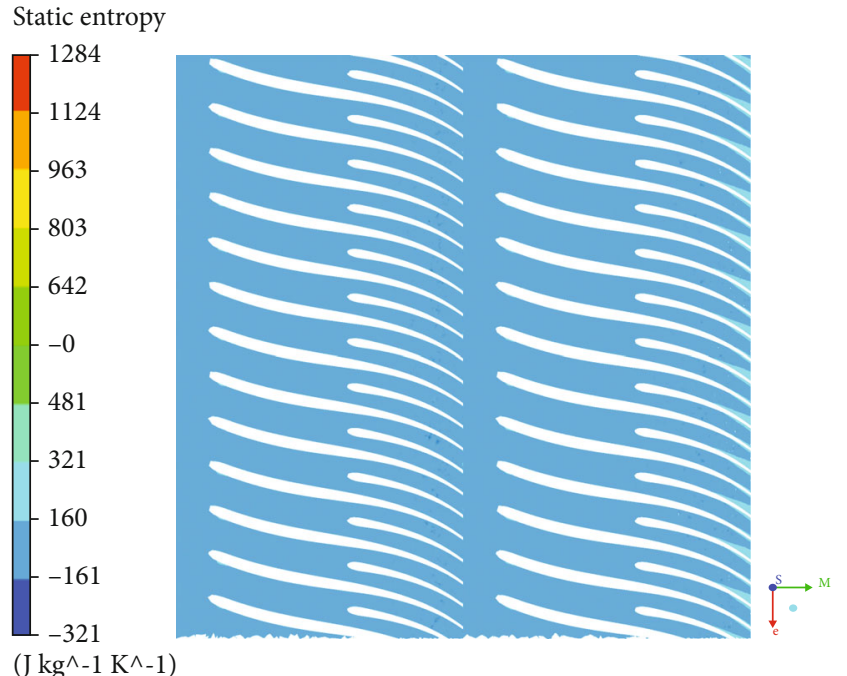

(a)

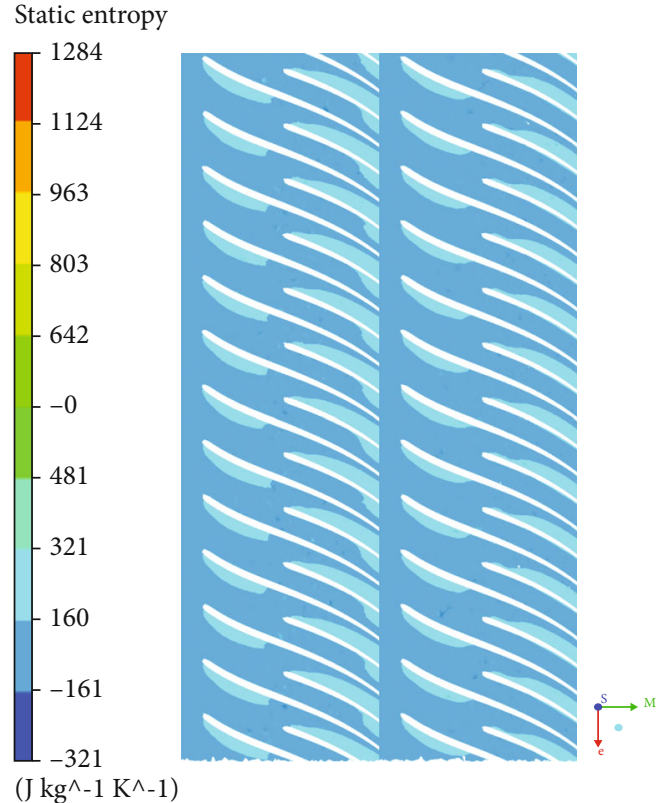

(b)

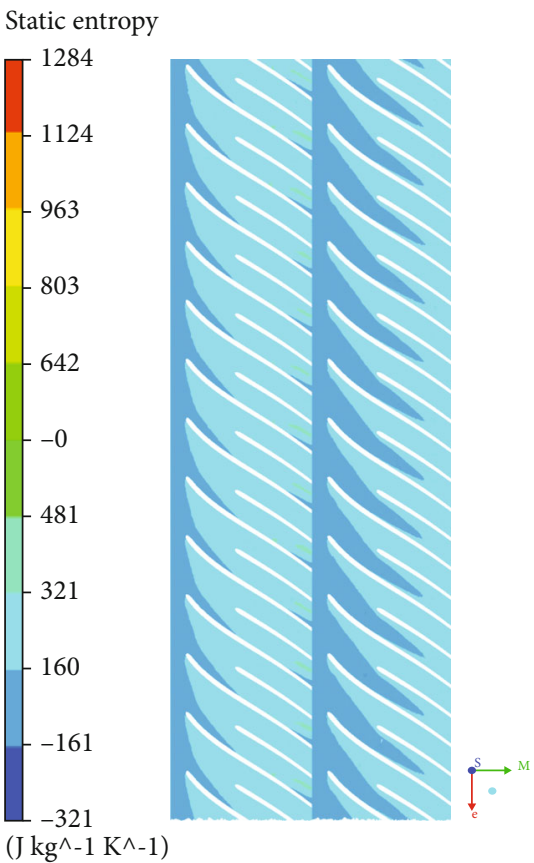

(c)

Figure 18: Blade-to-blade planes of double-sided centrifugal compressor for static entropy. (a) 0.1 span. (b) 0.5 span. (c) 0.9 span.

TABLE 7: Parameters of each section during compression.

\begin{tabular}{lccc}
\hline \multirow{2}{*}{ Parameters } & \multicolumn{3}{c}{ Section position } \\
& Section 1 & Section 2 & Section 3 \\
\hline Total pressure $P_{t}(\mathrm{~Pa})$ & 101325 & 293984 & 603569 \\
Total temperature $T_{t}(\mathrm{~K})$ & 288 & 407.989 & 539.455 \\
Air mass flow $W_{a}(\mathrm{~kg} / \mathrm{s})$ & 0.84 & 0.84 & 0.84 \\
Total pressure ratio $\pi_{c}{ }^{*}$ & & 2.901 & 2.053 \\
Isentropic efficiency $\eta_{c}{ }^{*}$ & & 0.8538 & 0.7081 \\
\hline
\end{tabular}

and the cooling effect is evident at the middle and root in the turbine. This shows that the high-pressure gas flows along the root to the tip of the turbine inside and impulsively cools the turbine wall. On the other hand, since the outlet velocity of the throat of the turbine outside is high, the cooling effect is also good. The cooling effect is not evident far away from the high-pressure gas.

In addition, the maximum temperature of the turbine wall is only $840.35 \mathrm{~K}$, which is far below the maximum temperature limit of the GH93 of $1088 \mathrm{~K}$. This means that it not only provides the potential for increasing the 


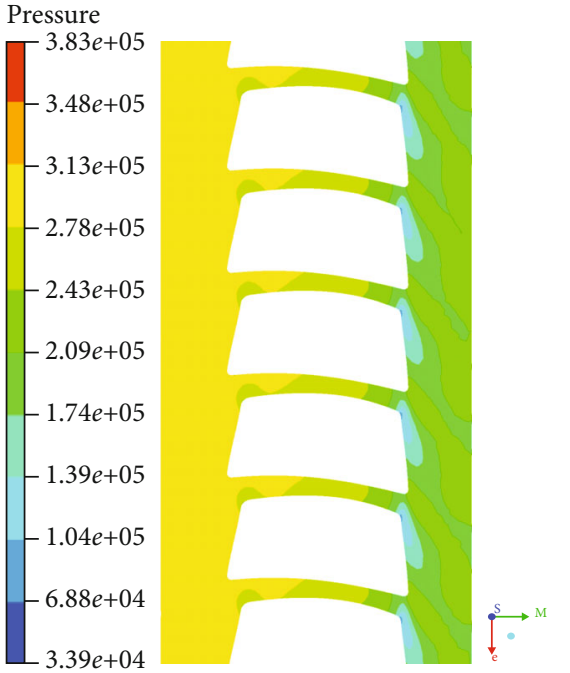

$(\mathrm{Pa})$

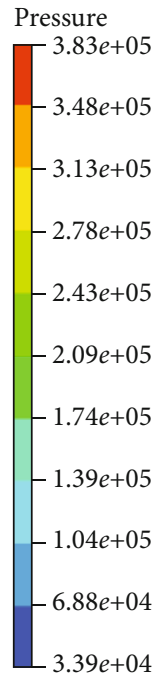

$(\mathrm{Pa})$

(a)

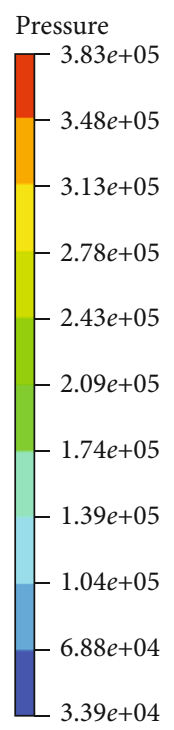

(Pa)

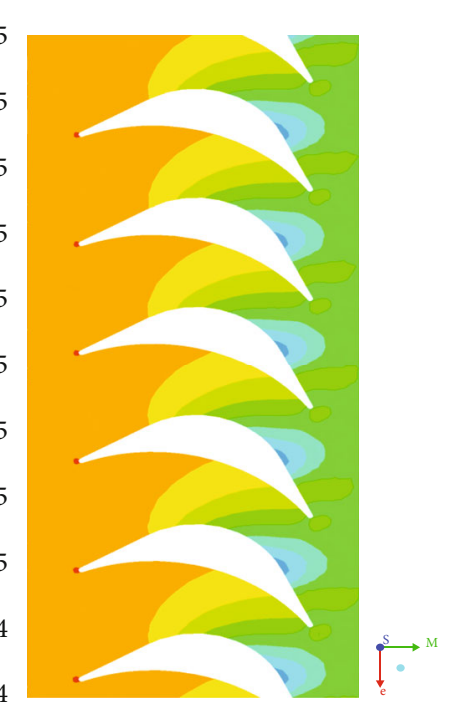

(d)

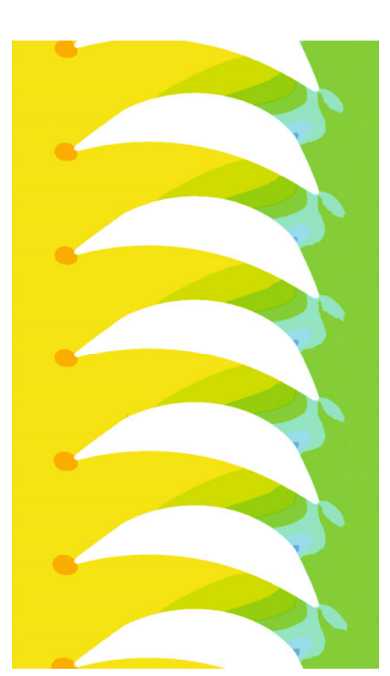

(b)

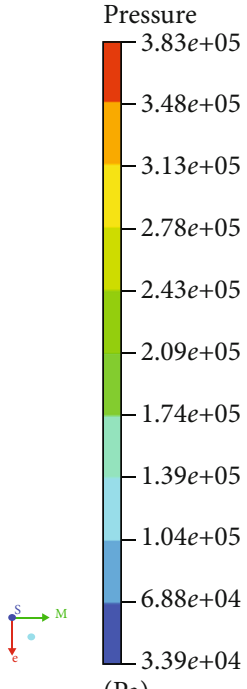

$(\mathrm{Pa})$

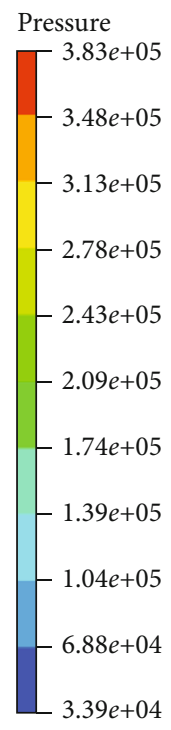

$(\mathrm{Pa})$

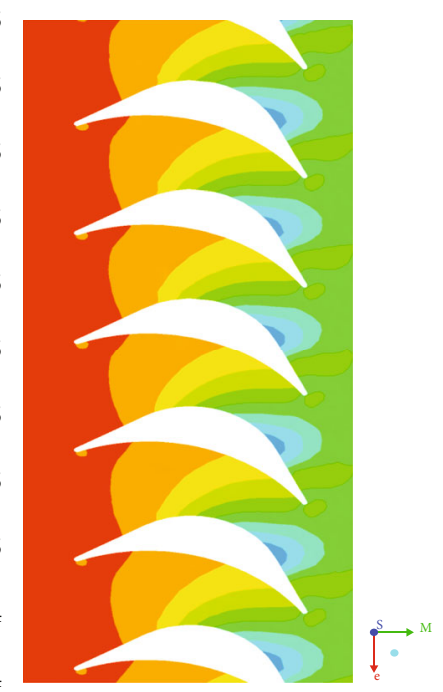

(e)

Figure 19: Static pressure contours of turbine channel. (a) 0.1 span. (b) 0.3 span. (c) 0.5 span. (d) 0.7 span. (e) 0.9 span.

turbine inlet total temperature but also provides room for development for the use of low-temperature, low-cost, and lightweight materials, such as titanium alloys and highentropy alloys. From the perspective of lightweight materials, this shows that the structural weight of the engine can be further reduced, which is conducive to the improvement of $T W R$.

First, based on the previous assumption, the hollow turbine guide is simplified to the absolute inlet velocity boundary condition of the turbine. On the other hand, there is a certain total pressure loss in the actual hollow turbine guide, which cannot be ignored. The total pressure recovery coefficient of the hollow turbine guide $\sigma_{4 \mathrm{~B}}$ is 0.972 , and the hollow turbine guide outlet total pressure $P_{t 4 \mathrm{~B}}$ is $574703 \mathrm{~Pa}$, as calculated by

$$
P_{t 4 B}=\sigma_{4 B} P_{t 4}
$$

Second, the total pressure drop ratio of the turbine $\pi_{T}{ }^{*}$ is 2.532, and the isentropic efficiency $\eta_{T}{ }^{*}$ is 0.9088 based on numerical simulation. The results show that a turbine with a blade shroud can avoid tip leakage to a certain extent and improve the isentropic efficiency.

Based on the above analysis, Table 8 shows the crosssectional parameters of the expansion process. The turbine power $W_{T}$ is $249.74 \mathrm{~kJ} / \mathrm{kg}$ and can be expressed as

$$
W_{T}=\frac{k^{\prime}}{k^{\prime}-1} R^{\prime} T_{t 4}\left(1-\frac{1}{\pi_{T}{ }^{*}\left(k^{\prime}-1\right) / k^{\prime}}\right) \eta_{T}{ }^{*} .
$$




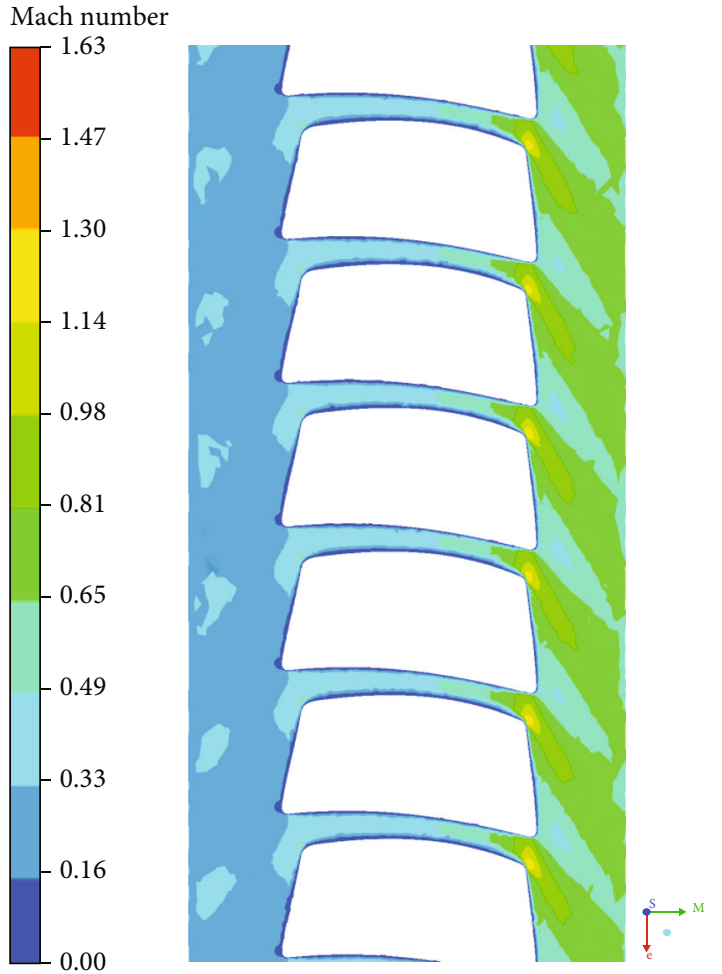

(a)

Mach number

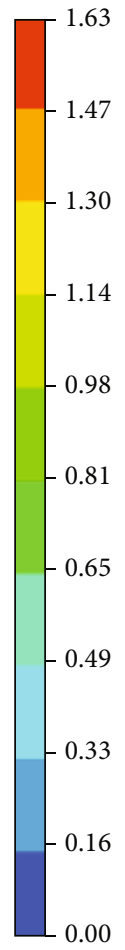

(c)

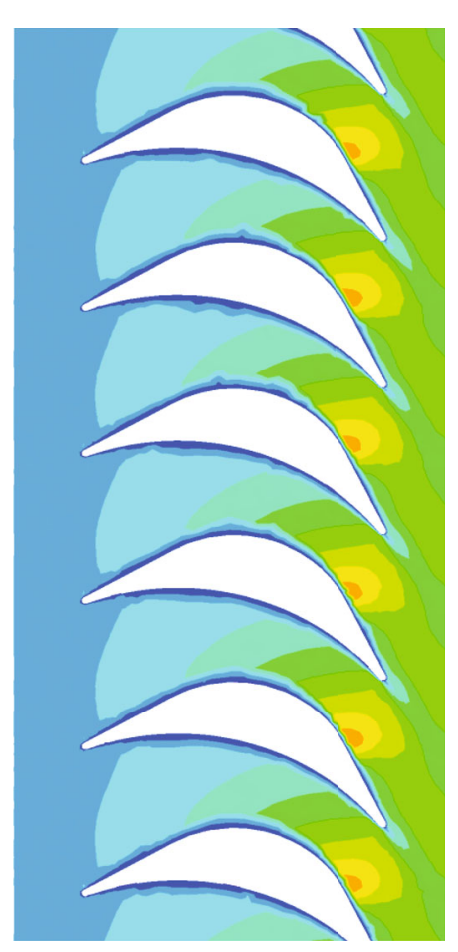

Mach number
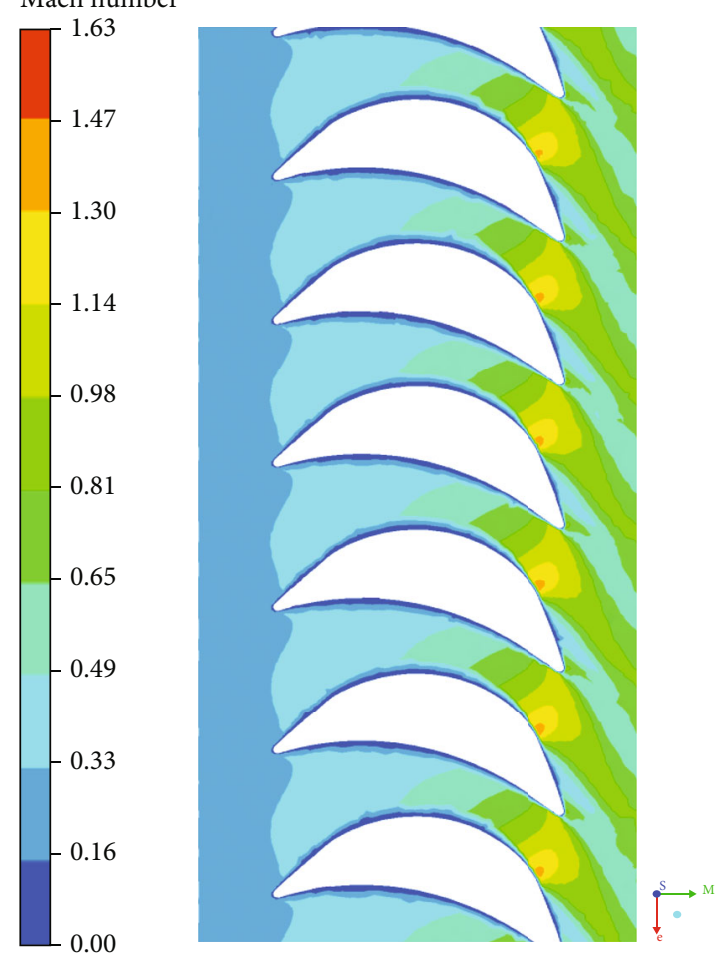

(b)

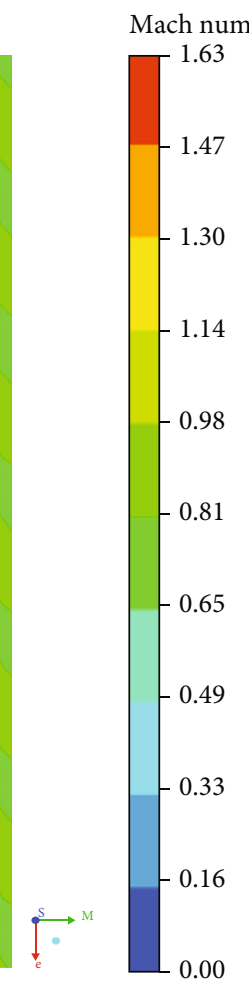

Mach number

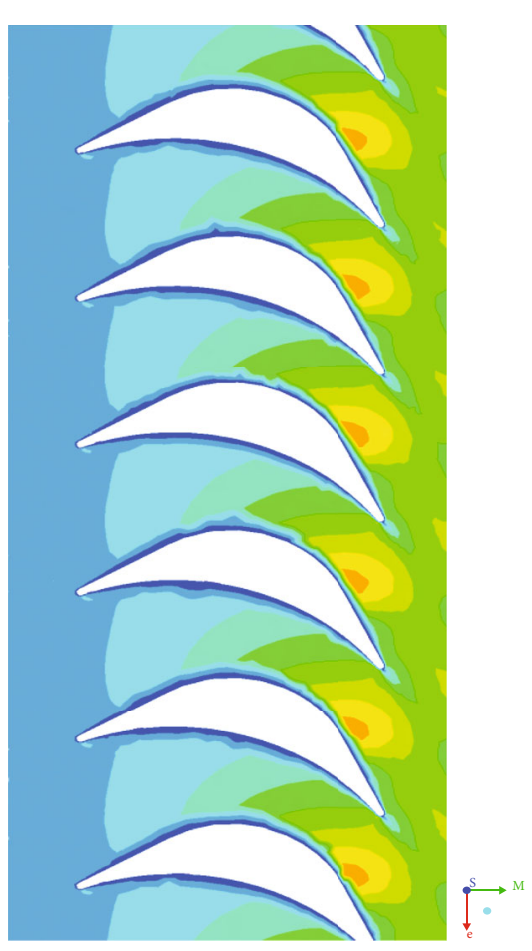

(d)

Figure 20: Continued. 


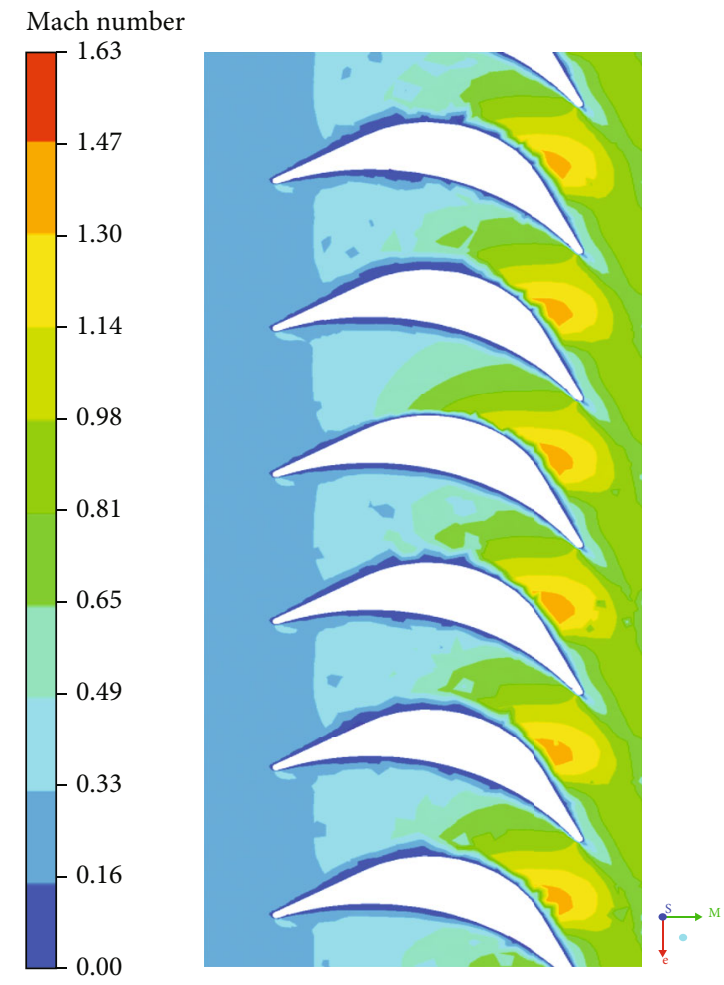

(e)

Figure 20: Relative Mach number contours of turbine channel. (a) 0.1 span. (b) 0.3 span. (c) 0.5 span. (d) 0.7 span. (e) 0.9 span.

Finally, the compressor power $P_{C}$ is $212.2 \mathrm{~kW}$, and the turbine power $P_{T}$ is $212.2 \mathrm{~kW}$ as calculated by formulas (12) and (13). This means that the turbine power can meet the compression needs of the DSCI.

$$
\begin{aligned}
& P_{C}=W_{k} W_{a}, \\
& P_{T}=W_{T} W_{g} \eta_{m},
\end{aligned}
$$

where the mechanical efficiency $\eta_{m}$ is 0.995 .

Through the previous calculations, all components met the matching characteristics in the gas generator. Indispensably, according to the outlet parameters of the turbine, the performance and economic indicators of the DSCI jet engine can be analyzed and compared with the JetCat P500-PRO$\mathrm{GH}$ of the same radial size.

First, the nozzle working status needs to be judged in the above hypothesis. Regarding the nozzle total pressure recovery coefficient $\sigma_{n z}$, considering the loss along the nozzle and the total pressure loss caused by the rear intake pipe to the rectification of the turbine exhaust gas, the empirical coefficient $\sigma_{n z}$ is 0.95 .

Second, the critical pressure drop ratio of the nozzle $\pi_{c r, n z}$ is 1.947 , and the available pressure drop ratio $\pi_{u s, n z}$ is 2.128 , as calculated by

$$
\pi_{c r, n z}=1.85 / \sigma_{n z}
$$

$$
\pi_{u s, n z}=P_{t 5} / P_{H}
$$

where $P_{H}$ is $101325 \mathrm{~Pa}$.

Comparing the critical pressure drop ratio and available pressure drop ratio, $\pi_{u s, n z}$ is greater than $\pi_{c r, n z}$. This means that the nozzle is in a working supercritical state. Additionally, the gas is not fully expanded at the outlet of the nozzle, which is characterized by the local speed of sound at the outlet of the nozzle, and the outlet static pressure is greater than the outside atmospheric pressure.

The outlet velocity of the nozzle $C_{6}$ is $537.74 \mathrm{~m} / \mathrm{s}$ as calculated by the following formula:

$$
\begin{aligned}
C_{6} & =\phi_{n z} \sqrt{\frac{2 k^{\prime}}{k^{\prime}-1} R^{\prime} T_{t 5}\left[1-\frac{1}{\left(\sigma_{n z} P_{t 5} / P_{9}\right)\left(k^{\prime}-1\right) / k^{\prime}}\right]} \\
& =\phi_{n z} \sqrt{\frac{2 k^{\prime}}{k^{\prime}+1} R^{\prime} T_{t 5},}
\end{aligned}
$$

where the speed factor $\varphi_{n z}$ is 0.97 .

In addition, the outlet cross-sectional area of the nozzle $A_{6}$ is $3.05 \times 10^{-3} \mathrm{~m}^{2}$, as calculated by

$$
A_{6}=\frac{W_{g} \sqrt{T_{t 6}}}{K_{m}{ }^{\prime} P_{t 6} q\left(\lambda_{6}\right)},
$$

Since the flow process of the nozzle is an isentropic flow, the nozzle inlet total temperature $T_{t 6}$ is equal to outlet total 


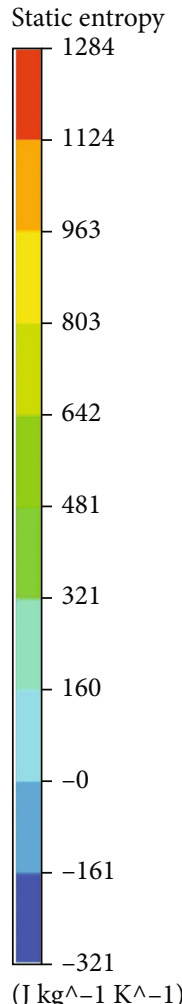

$\left(\mathrm{J} \mathrm{kg} \wedge-1 \mathrm{~K}^{\wedge}-1\right)$

Static entropy

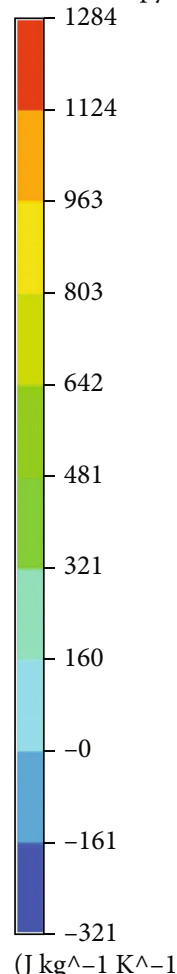

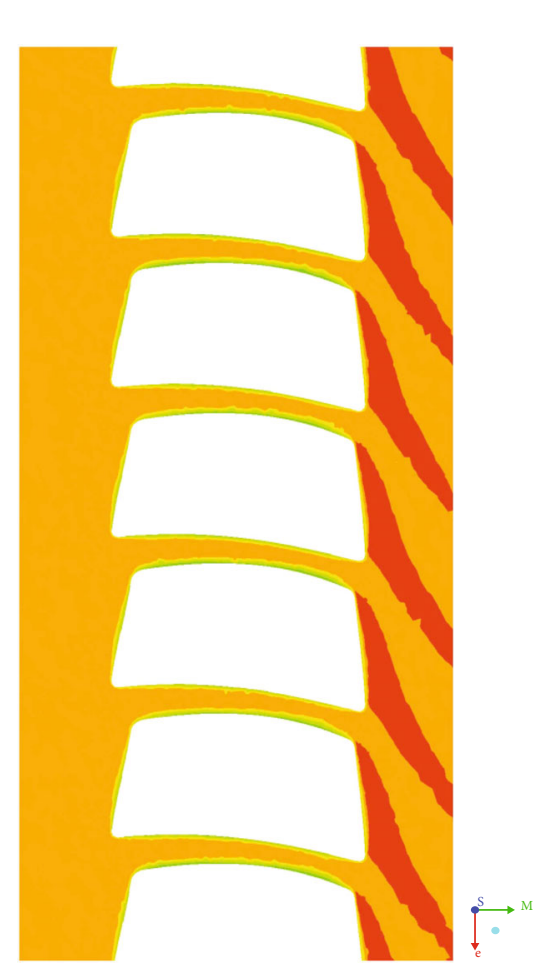

(a)

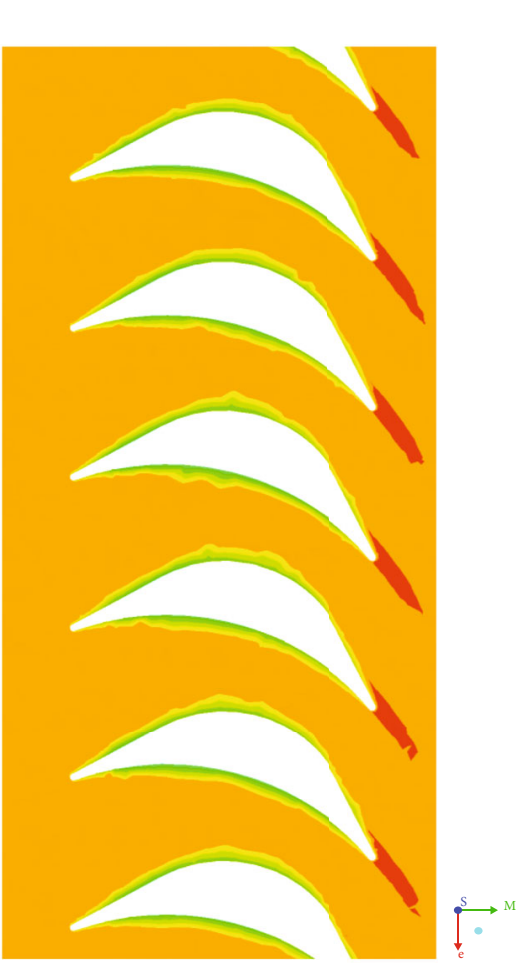

(c)

Static entropy

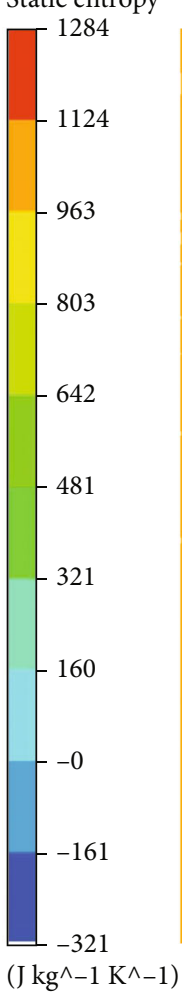

$\left(\mathrm{J} \mathrm{kg} \wedge-1 \mathrm{~K}^{\wedge}-1\right)$

Static entropy

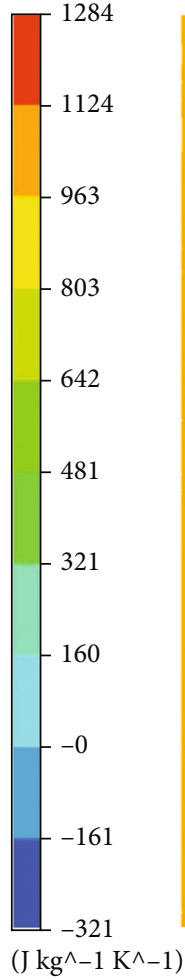

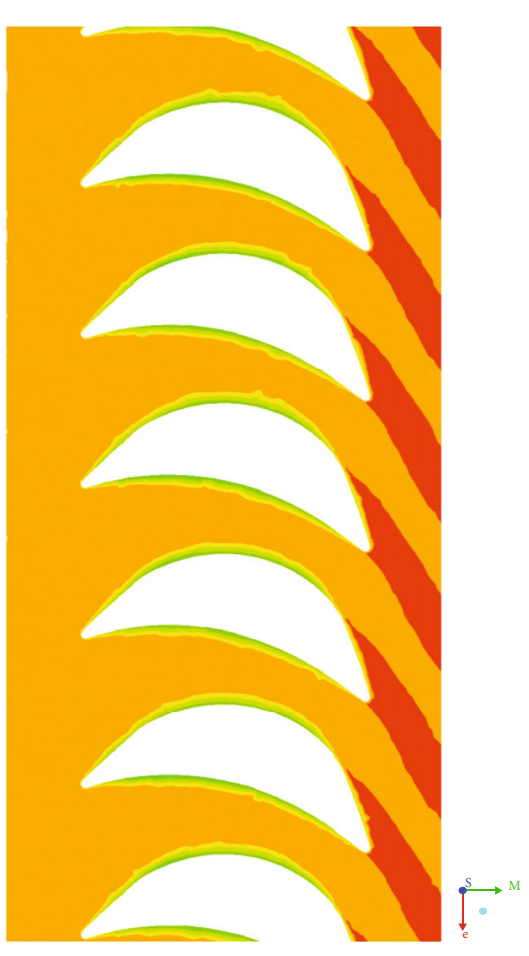

(b)

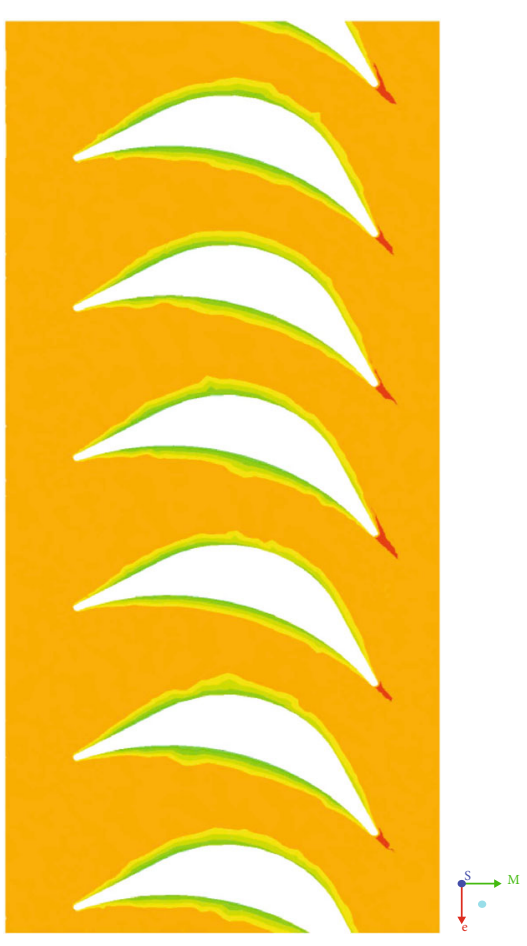

(d)

FIgUre 21: Continued. 


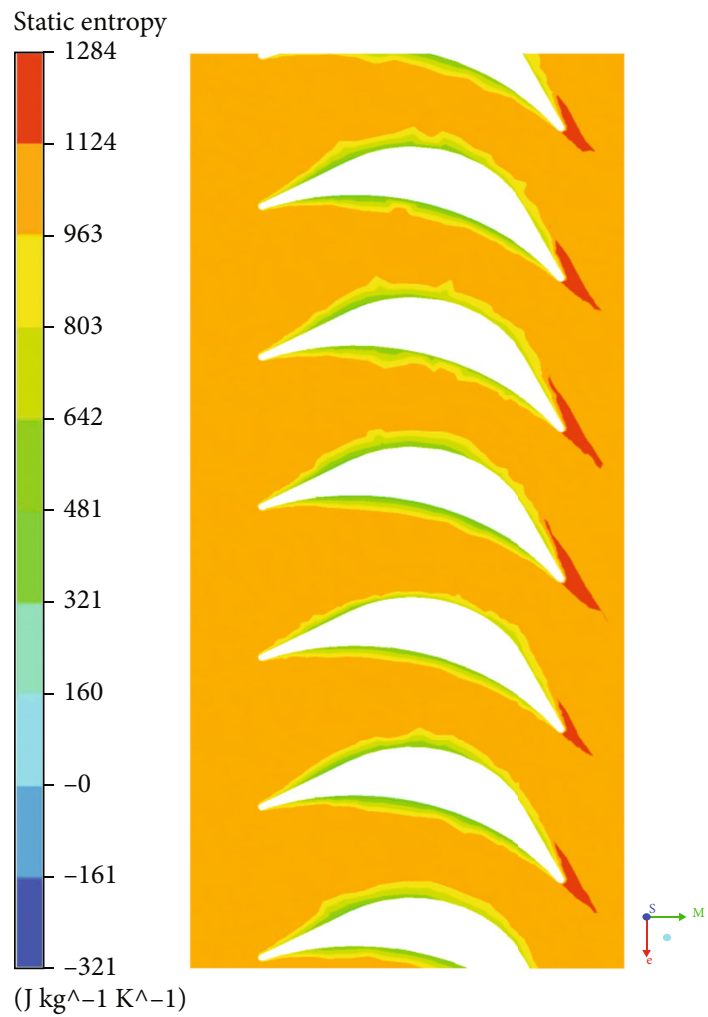

(e)

FIgURE 21: Static entropy contours of turbine channel. (a) 0.1 span. (b) 0.3 span. (c) 0.5 span. (d) 0.7 span. (e) 0.9 span.

temperature $T_{t 5}$; the nozzle outlet relative dense flow $q(\lambda 6)$ is 1 ; and $K_{m}{ }^{\prime}$ is 0.0397 .

The nozzle outlet static pressure $P_{6}$ is $116535 \mathrm{~Pa}$, as calculated by

$$
P_{6}=\frac{P_{t 6}}{\left(1+\left(\left(k^{\prime}-1\right) / 2\right) M_{6}{ }^{2}\right)^{k^{\prime} /\left(k^{\prime}-1\right)}},
$$

where the nozzle outlet Mach number $M_{6}$ is 1 .

Through the parameters derived above, the performance and economic indicators of the DSCI jet engine can be calculated, as shown in the following formulas:

The DSCI jet engine thrust can be expressed as

$$
F=W_{g} C_{6}-W_{a} V+\left(P_{6}-P_{H}\right) A_{6} .
$$

The DSCI jet engine thrust $F$ is $505.62 \mathrm{~N}$ when the flight speed $V$ is 0 .

The DSCI jet engine specific thrust can be expressed as

$$
F_{s}=\frac{F}{W_{a}} .
$$

The DSCI jet engine specific thrust $F_{s}$ is $601.93(\mathrm{~N} \mathrm{~s}) / \mathrm{kg}$.
The DSCI jet engine fuel consumption rate can be expressed as

$$
s f c=\frac{3600 m_{f}}{F} .
$$

The DSCI jet engine fuel consumption rate $s f c$ is 9.54\% [13].

Table 9 shows the important parameters of the DSCI jet engine and the JetCat P500-PRO-GH considering the design parameters. Compared to the JetCat P500-PRO-GH, the DSCI jet engine has a $34.37 \%$ reduction in axial size and a $13.49 \%$ reduction in weight due to its compact structure. Although there is a certain reduction in mass flow relative to the JetCat P500-PRO-GH, with the substantial increase in OPR (close to the optimal supercharging ratio) and turbo efficiency, the DSCI jet engine has a slightly higher in thrust than the JetCat engine. Additionally, the specific thrust increased by $10.11 \%$.

Under the combined effect of various parameters, the $T W R$ of the DSCI jet engine is increased to 12.17 , which is $39.4 \%$ higher than that of the JetCat P500-PRO-GH. In addition, the increased $O P R$ is also close to the optimal economic pressure ratio. Coupled with the phenomenon of convective heat exchange inside and outside the turbine and the recovery of exhaust gas heat, the DSCI engine has a $36.82 \%$ reduction in $s f c$.

Based on the above description, the DSCI jet engine has significant advantages in performance indicators and 


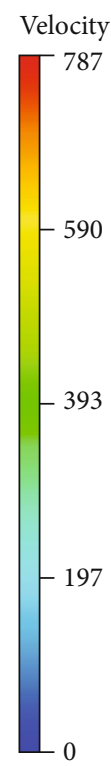

$\left(\mathrm{m} \mathrm{s}^{\wedge}-1\right)$

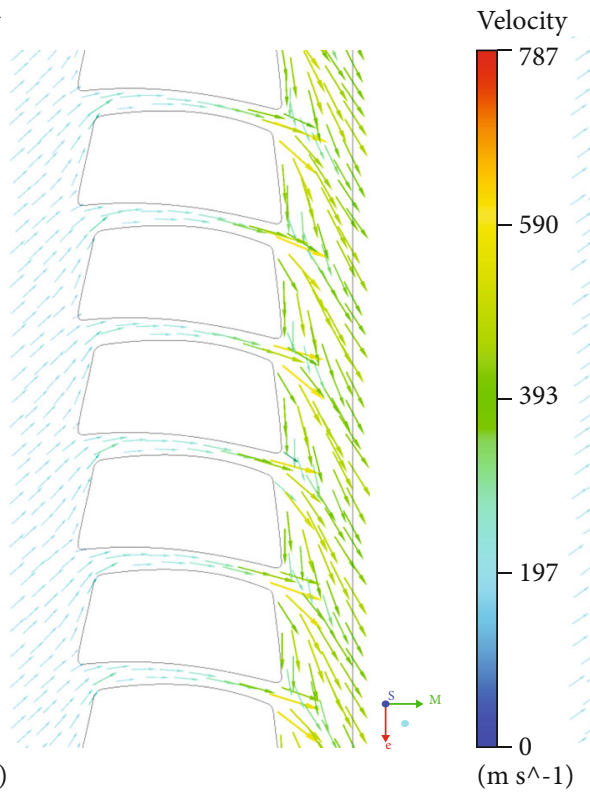

(a)

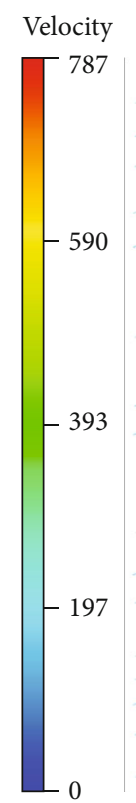

$\left(\mathrm{m} \mathrm{s}^{\wedge}-1\right)$

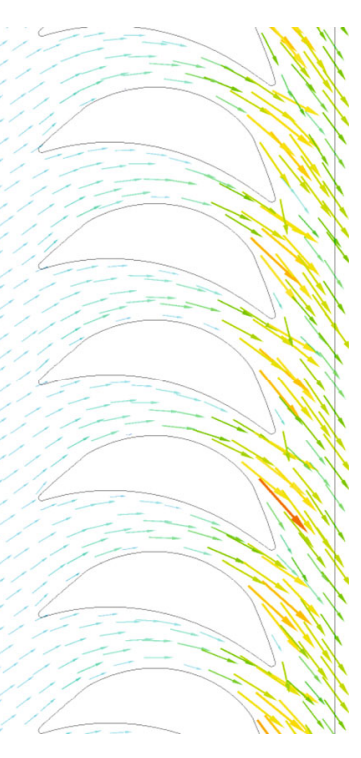

(b)
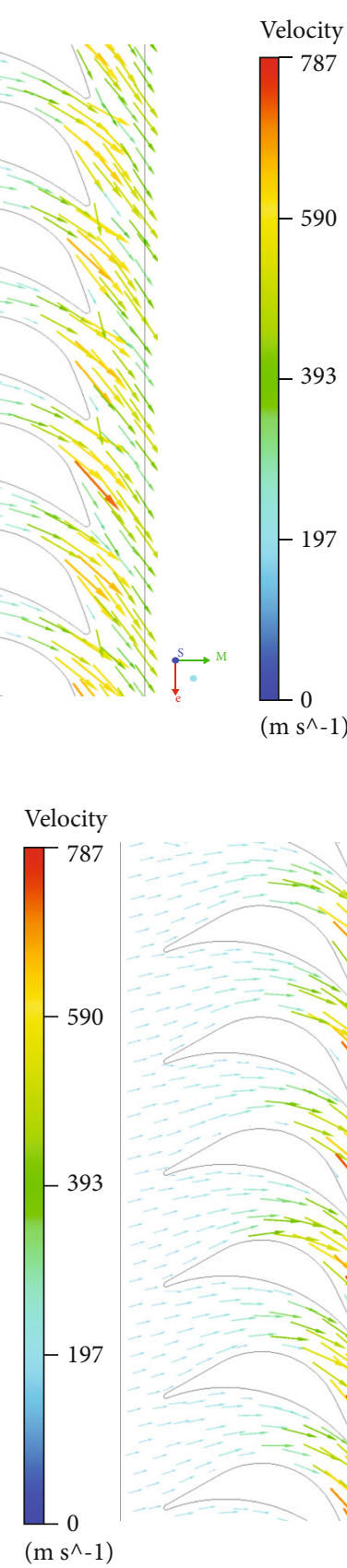

(d)

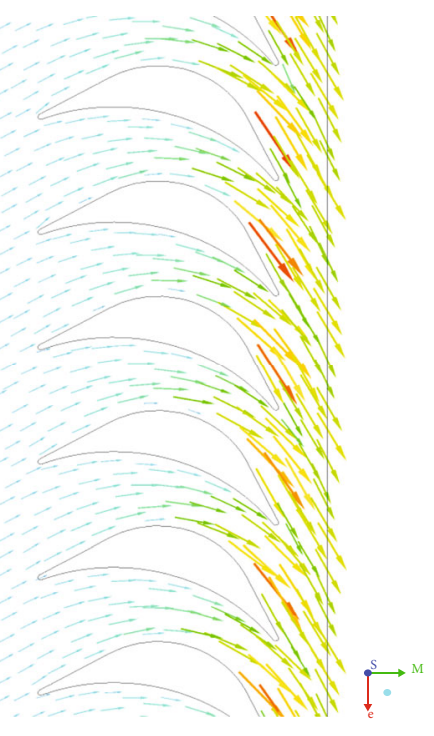

(c)

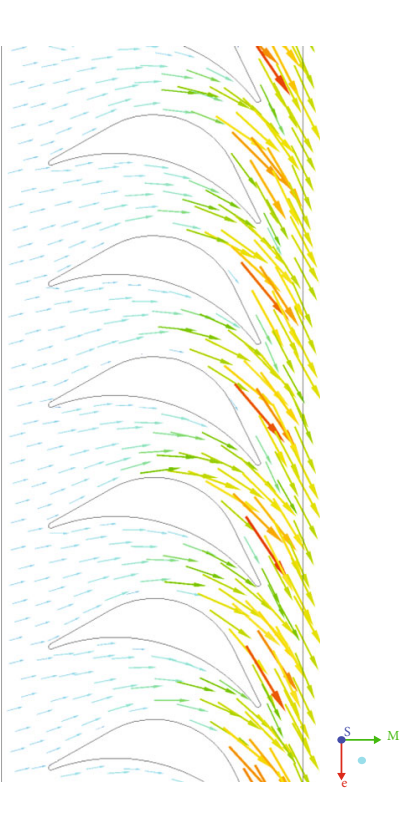

(e)

FIGURE 22: Relative velocity vector contours of turbine channel. (a) 0.1 span. (b) 0.3 span. (c) 0.5 span. (d) 0.7 span. (e) 0.9 span. 


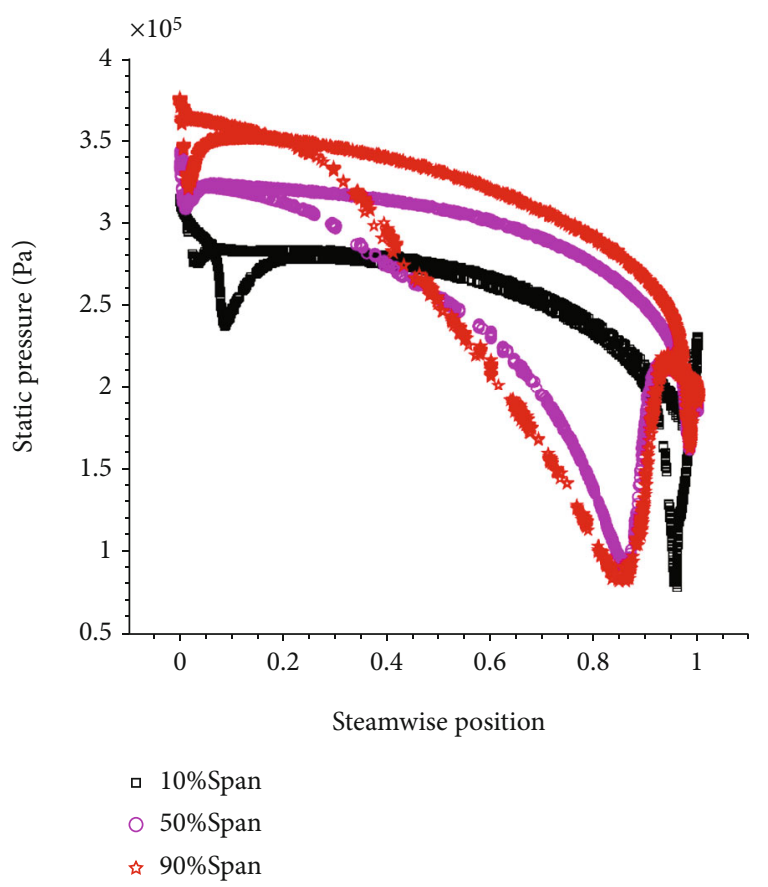

FIGURE 23: Static pressure distribution of turbine ( $0 \%$ represents the hub; $100 \%$ indicates the tip).

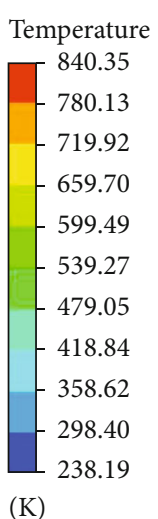

(K)

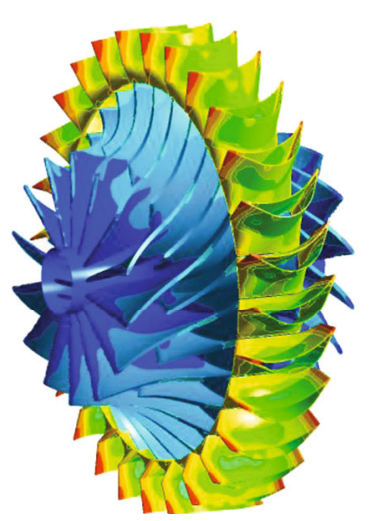

(a)

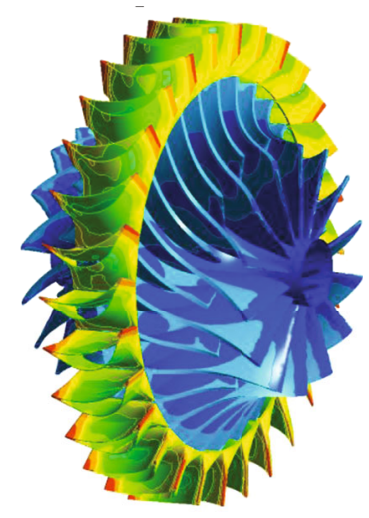

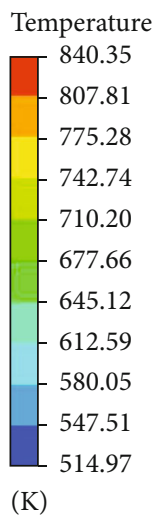

$(\mathrm{K})$
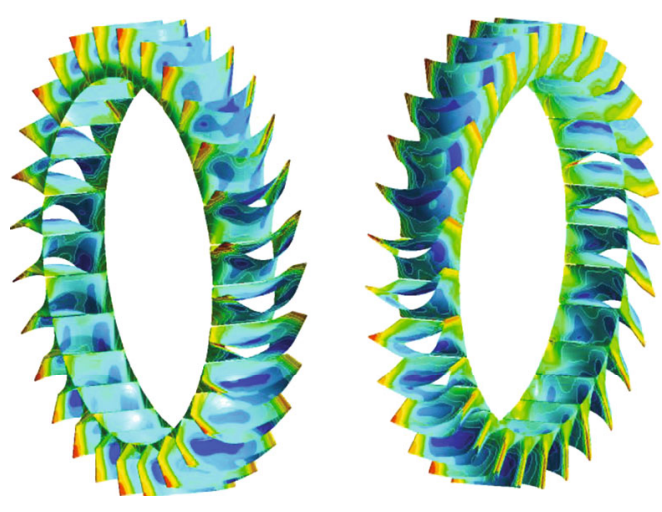

(b)

Figure 24: Static temperature field contours. (a) Static temperature field contour of the DSCI. (b) Static temperature field contour the turbine outside.

TABLE 8: Parameters of each section during the expansion process.

\begin{tabular}{lccc}
\hline Parameters & Section 4 & Section position & Section 4B \\
Total pressure $P_{t}(\mathrm{~Pa})$ & 591498 & 574703 & Section 5 \\
Total temperature $T_{t}(\mathrm{~K})$ & 1150 & 1150 & 227010 \\
Gas flow $W_{g}(\mathrm{~kg} / \mathrm{s})$ & 0.854 & 0.854 & 934.731 \\
Total pressure drop ratio $\pi_{T}{ }^{*}$ & & & 0.854 \\
Isentropic efficiency $\eta_{T}{ }^{*}$ & & & 2.532 \\
\hline
\end{tabular}


TABLE 9: Design point parameters of the DSCI jet engine and JetCat P500-PRO-GH.

\begin{tabular}{lcc}
\hline Parameters & & Engine type \\
& DSCI jet engine & JetCat P500-PRO-GH \\
\hline Diameter $d(\mathrm{~mm})$ & 178 & 178.6 \\
Length $L(\mathrm{~mm})$ & 275 & 419 \\
Weight $G(\mathrm{~g})$ & 4239 & 4900 \\
Mass flow $W_{a}(\mathrm{~kg} / \mathrm{s})$ & 0.84 & 0.9 \\
Maximum rotating speed $n_{\max }(\mathrm{r} / \mathrm{min})$ & 80000 & 80000 \\
OPR & 5.957 & 3.6 \\
Exhaust total temperature $T_{t 5}(\mathrm{~K})$ & 934.731 & 1013 \\
Exhaust speed $C_{6}(\mathrm{~m} / \mathrm{s})$ & 537.74 & 551.67 \\
Thrust $F(\mathrm{~N})$ & 505.62 & 492 \\
TWR & 12.17 & 8.73 \\
Maximum fuel consumption rate $s f c(\mathrm{~kg} /(\mathrm{Nh}))$ & 0.0954 & 0.151 \\
Specific thrust $F_{s}(\mathrm{~N} \mathrm{~s}) / \mathrm{kg}$ & 601.93 & 546.67 \\
\hline
\end{tabular}

economic indicators in the same size radial engine, especially the potential to increase the turbine inlet total temperature and the complete expansion of the nozzle, which can make the DSCI jet engine performance higher.

\section{Conclusion}

This research presents a conceptual design of DSCI for a high-TWR propulsion system. Through the work discussed above, the following conclusions can be drawn:

(1) The DSCI engine includes the DSCI jet engine scheme and the gas-driven propeller DSCI engine scheme. In theory, compared with the traditional MTEs, the DSCI engine has good shock absorption and response characteristics. And its structural weight reduction and heat recovery provide imagination for MTE's performance improvement. On the other hand, with the help of additive manufacturing technology, DSCI jet engine installation is more convenient and the structure is more reliable

(2) After satisfying four balance conditions and several assumptions, the turbine must adopt the design rules of skewed blades and transition sections. Preliminary design of the DCSI rotor, in which the profile of the turbine outside adopts a traditional turbine and the turbine inside adopts a tubular diffuser structure

(3) The thermal analysis of different processes shows that all components meet the matching characteristics of the gas generators at the design point. Among them, the OPR of the compression process $\pi_{k}{ }^{*}$ is 5.957, and the overall isentropic efficiency $\eta_{k}{ }^{*}$ is 0.7617 ; the total pressure drop ratio of the turbine $\pi_{T}{ }^{*}$ is 2.532, and the isentropic efficiency $\eta_{T}{ }^{*}$ is 0.9088. Notably, the cooling characteristics of the turbine, the high-pressure gas inside the turbine, and the high-temperature gas outside the turbine convectively exchange heat, so that the maximum temperature of the turbine drops to $840.35 \mathrm{~K}$, which is much lower than the maximum temperature limit of the GH93 of $1088 \mathrm{~K}$. These characteristics provide room for the development of an increased turbine inlet total temperature or the use of low-cost and lightweight materials such as titanium alloys. From the perspective of lightweight materials, this means that the structural weight can be further reduced, which is conducive to the improvement of DSCI jet engine TWR

(4) Compared to the JetCat P500-PRO-GH, the DSCI jet engine has a $34.37 \%$ reduction in axial size and a $13.49 \%$ reduction in weight. Due to structural weight reduction, high $O P R$, and exhaust heat recovery, the TWR is increased by $39.4 \%$, and the $s f c$ is reduced by $36.82 \%$. So, the DSCI jet engine has significant advantages in performance indicators and economic indicators in the same size engine, especially the potential to increase the turbine inlet total temperature and the complete expansion of the nozzle, which can make the DSCI jet engine performance higher.

\section{Data Availability}

The data used to support the findings of this study are included within the article.

\section{Conflicts of Interest}

The authors declare no conflicts of interest.

\section{Acknowledgments}

This study was supported by the Jiangxi Province Graduate Student Innovation Special Fund Project (YC2019-S342) and the National Natural Science Foundation of China (No. 51766011). 


\section{References}

[1] O. Dessornes, S. Landais, R. Valle et al., "Advances in the development of a microturbine engine," Journal of Engineering for Gas Turbines and Power-Transactions of the Asme, vol. 136, no. 7, p. 9, 2014.

[2] N. Chigier and T. Gemci, "A review of micro propulsion technology," in 41st Aerospace Sciences Meeting and Exhibit, Reno, Nevada, January 2003.

[3] N. Marin and P. Spătaru, "The role and importance of UAV within the current theaters of operations," INCAS Bulletin, vol. 2, no. 2, pp. 66-74, 2010.

[4] F. Nicolosi, P. Della Vecchia, and S. Corcione, "Design and aerodynamic analysis of a twin-engine commuter aircraft," Aerospace Science and Technology, vol. 40, pp. 1-16, 2015.

[5] J. F. Rideau, G. Guyader, and A. Cloarec, "Microturbo families of turbojet engine for missiles and UAV's from the TR60 to the new bypass turbojet engine generation," in Aiaa/asme/sae/asee Joint Propulsion Conference \& Exhibit, Paper 2008-4590, Hartford, CT, June 2013.

[6] M. Harris, A. Jones, and E. Alexander, "Miniature turbojet development at Hamilton Sundstrand the TJ-50, TJ-120 and TJ-30 turbojets," in 2nd AIAA "Unmanned Unlimited" Conf. and Workshop \& Exhibit, San Diego, California, September 2003.

[7] J. Higashi, S. Nakagami, K. Matsuoka et al., "Experimental study of disk-shaped rotating detonation turbine engine," in 55th AIAA Aerospace Sciences Meeting, Grapevine, Texas, January 2017.

[8] R. T. Huff, S. A. Boller, M. D. Polanka, F. R. Schauer, M. L. Fotia, and J. L. Hoke, "Radial rotating detonation engine driven bleed air turbine," Journal of Propulsion and Power, vol. 37, no. 2, pp. 252-260, 2021.

[9] P. Akbari and M. D. Polanka, "Performance of an ultracompact disk-shaped reheat gas turbine for power generation," in 2018 Joint Propulsion Conference, Cincinnati, Ohio, July 2018.

[10] Y. Lu, A. P. Roskilly, X. Yu, L. Jiang, and L. Chen, “Technical feasibility study of scroll-type rotary gasoline engine: a compact and efficient small-scale Humphrey cycle engine," Applied Energy, vol. 221, pp. 67-74, 2018.

[11] C. Zhang, Y. Wang, K. Wang, J. W. Wang, and S. F. He, "Compression performance of double-sided composite impeller with micro gas turbine," Journal of Aerospace Power, vol. 35, pp. 1042-1050, 2020

[12] C. Zhang and Y. Wang, "A miniature turbojet engine with double-sided new composite impeller," 2019, Chinese patent.

[13] B. Luo, C. Zhang, and Y. Wang, "A double-sided composite impeller turbine engine based on air-driven blades," 2021, Chinese patent.

[14] W. Y. Lu, G. P. Huang, X. Xiang, J. Wang, and Y. Yang, "Thermodynamic and aerodynamic analysis of an air-driven fan system in low-cost high-bypass-ratio turbofan engine," Energies, vol. 12, no. 10, p. 1917, 2019.

[15] Q. Du and Z. B. Yuan, Aircraft engine design manual, vol. 8, Aviation Industry Press, Beijing, 2st edition, 2000.

[16] L. M. He, Principles of Aircraft Propulsion System, National Defense Industry Press, Beijing, 2st edition, 2017.

[17] D. P. Kenny, “A comparison of the predicted and measured performance of high pressure ratio centrifugal compressor diffusers," in ASME 1972 International Gas Turbine and Fluids
Engineering Conference and Products Show, San Francisco, California, USA, March 1972.

[18] H. Li and D. G. Huang, "Aerodynamic optimization design of a multistage centrifugal steam turbine and its off-design performance analysis," International Journal of Rotating Machinery, vol. 2017, Article ID 4690590, 11 pages, 2017.

[19] K. Kusterer, D. Bohn, T. Sugimoto, and R. Tanaka, "Conjugate calculations for a film-cooled blade under different operating conditions," in Proceedings of the ASME Turbo Expo 2004: Power for Land, Sea, and Air. Volume 3: Turbo Expo 2004, pp. 675-684, Vienna, Austria, January 2004.

[20] Z. X. Han, B. H. Dennis, and G. S. Dulikravich, "Simultaneous prediction of external flow-field and temperature in internally cooled 3-D turbine blade material," in Proceedings of the ASME Turbo Expo 2000: Power for Land, Sea, and Air. Volume 3: Heat Transfer; Electric Power; Industrial and Cogeneration, Munich, Germany, May 2000.

[21] Wrought Superalloys, C. s. E. C. o. C. A. M. H., China Aeronautical Materials Handbook, vol. 2, China Standard Press, Beijing, 2st edition, 2001.

[22] V.-M. Lei, "Aerodynamics of a centrifugal compressor with a double sided impeller," in Proceedings of the ASME 2011 Turbo Expo: Turbine Technical Conference and Exposition. Volume 7: Turbomachinery, Parts A, B, and C, Vancouver, British Columbia, Canada, May 2012.

[23] R. Yang, G. Wang, H. Liu, H. Xiang, and J. Gao, "Numerical study on the flow mechanism of compressor rotor blade vibration under different inlet probe configurations," International Journal of Aerospace Engineering, vol. 2020, Article ID 8897211, 12 pages, 2020.

[24] G. Wang, N. Ge, and D. Zhong, "Numerical investigation of the wake vortex-related flow mechanisms in transonic turbines," International Journal of Aerospace Engineering, vol. 2020, Article ID 8825542, 18 pages, 2020.

[25] C. Cui, Z. Zhou, and E. Liu, "Aerodynamic optimization design of a supersonic compressor rotor with high pressure ratio," International Journal of Aerospace Engineering, vol. 2021, Article ID 6664968, 24 pages, 2021.

[26] Y. Wang, Principles of Aero Engines, Beijing University of Aeronautics and Astronautics Press, Beijing, 1st edition, 2009. 